\title{
From Explicit to Implicit Density Functionals
}

\author{
E. ENGEL, R. M. DREIZLER \\ Institut für Theoretische Physik, Universität Frankfurt, Robert-Mayer-Strasse 8-10, D-60054, \\ Frankfurt am Main, Germany
}

Received 3 August, 1998; accepted 13 August 1998

\begin{abstract}
The concept of orbital- and eigenvalue-dependent exchangecorrelation (xc) energy functionals is reviewed. We show how such functionals can be derived in a systematic fashion via a perturbation expansion, utilizing the Kohn-Sham system as a noninteracting reference system. We demonstrate that the second-order contribution to this expansion of the xc-energy functional includes the leading term of the van der Waals interaction. The optimizedpotential method (OPM), which allows the calculation of the multiplicative xc-potential corresponding to an orbital- and eigenvalue-dependent xc-energy functional via an integral equation, is discussed in detail. We examine an approximate analytical solution of the OPM integral equation, pointing out that, for eigenvalue-dependent functionals, the three paths used in the literature for the derivation of this approximation yield different results. Finally, a number of illustrative results, both for the exchange-only limit and for the combination of the exact exchange with various correlation functionals, are given. (C) 1999 John Wiley \& Sons, Inc. J Comput Chem 20: 31-50, 1999
\end{abstract}

Keywords: density functional theory; exchange-correlation potential; optimized potential method; van-der-Waals forces; atomic correlation energies

\section{Introduction}

$\mathbf{T}$ he major task preceding the application of the Kohn-Sham (KS) method of density functional theory $(\mathrm{DFT})^{1-3}$ is the construction of a

Correspondence to: R. M. Dreizler

Contract/grant sponsor: Deutsche Forschungsgemeinschaft; contract grant number: Dr113/20-2. suitable approximation for the exchange-correlation (xc) energy functional, $E_{x c}[n]$, and the corresponding multiplicative xc-potential $v_{x c}([n] ; \mathbf{r})$. The xc-energy arises in the context of the mapping of the many-body problem onto a corresponding single-particle problem. Its definition is:

$$
E_{x c}=T-T_{s}+W-E_{H}
$$

$E_{x c}$ includes the difference between the full (interacting) kinetic energy, $T$, and the kinetic energy, 
$T_{s}$, of the auxiliary KS particles $(\hbar=1)$ :

$$
T_{s}=-\frac{1}{2 m} \sum_{\epsilon_{k} \leq \epsilon_{F}} \int d^{3} r \phi_{k}^{\dagger}(\mathbf{r}) \nabla^{2} \phi_{k}(\mathbf{r})
$$

and the difference between the full electron-electron interaction energy, $W$, and the Hartree energy:

$$
\begin{aligned}
E_{H} & =\frac{e^{2}}{2} \int d^{3} r \int d^{3} r^{\prime} \frac{n(\mathbf{r}) n\left(\mathbf{r}^{\prime}\right)}{\left|\mathbf{r}-\mathbf{r}^{\prime}\right|} \\
n(\mathbf{r}) & =\sum_{\boldsymbol{\epsilon}_{k} \leq \boldsymbol{\epsilon}_{F}} \phi_{k}^{\dagger}(\mathbf{r}) \phi_{k}(\mathbf{r})
\end{aligned}
$$

In the course of the development of DFT three distinct levels of approximations for $E_{x c}$ can be distinguished:

1) In the local density approximation (LDA) ${ }^{4}$ one uses the density dependence of the xcenergy density of a homogeneous electron gas and replaces the constant gas density by the actual, locally varying density $n(\mathbf{r})$. Initially, the input of the homogeneous system was derived by analysis and, to the extent possible, resummation of diagrammatic contributions to the ground-state energy. ${ }^{5,6}$ At a later stage, more accurate Green's function Monte-Carlo results ${ }^{7,8}$ were parameterized..$^{8-11}$

At first sight, the LDA seems to be too crude to deliver results of appropriate quality. It has, however, been found ${ }^{3,12,13}$ (and analyzed in some detail $1^{6,14,15}$ ) that, due to a cancelation of errors between exchange and correlation contributions and the compliance with an important sum rule for the xc-hole, the performance of the LDA is much better than could be expected, thus it is still a mainstay of the DFT-Ks applications. Nonetheless, the LDA involves definite errors, most notably the incomplete cancelation of the self-interaction energy in $E_{H}$ and the corresponding incorrect asymptotic behavior of the exchange potential for finite systems, which does not allow a correct description of atomic negative ions. ${ }^{16} \mathrm{~A}$ number of corrective measures, such as self-interaction correction, ${ }^{10,17,18}$ were devised to cope with these problems.

2) A natural extension of the LDA is the straightforward gradient expansion approximation (GEA), in which the lowest order deviations from homogeneity are taken into account on the basis of long-wavelength expansion of the linear response corrections to the homogeneous electron gas xc-energy. However, the rather extensive evaluation of the corresponding contributions ${ }^{19-26}$ did not lead to functionals which were successful in applications. The benefits of error cancelation between both exchange and correlation and between different regions in space, which save the LDA, are lost. In addition, the GEA no longer satisfies the sum rule for the xchole. For these reasons the positive features of the direct GEA were combined with semiempirical input ${ }^{27-29}$ or with corrective features incorporating properties of the pair correlation function ${ }^{30-34}$ to generate functionals that have been termed generalized gradient approximations (GGAs). With GGAs one is able to correct a number of the deficiencies of the LDA, such as, for instance, its prediction of a paramagnetic ground state for metallic iron. ${ }^{35}$ Moreover, these functionals have yielded excellent results for the structure and energetics of molecules. ${ }^{36-38}$ However, GGAs do not consistently improve the agreement with experiment (compared with the LDA), in particular for solids. ${ }^{39-44}$ For instance, the failure of the LDA to give the correct ground states for some transition metal oxides remains unresolved ${ }^{40}$ (although there might be some room left for improvement, ${ }^{43}$ if more emphasis is placed on the quality of the xc-potential rather than the xc-energy ${ }^{45}$ ). In addition, the GGA neither really improves the description of negative ions nor is able to deal with van der Waals bonds.

3) The third level in the search for improved ground-state energy functionals is the use of orbital-dependent representations. These constitute implicit functionals of the density, in the sense that the xc-functional is expressed in terms of KS orbitals (and KS eigenvalues, in general), rather than directly in terms of the density:

$$
\begin{gathered}
E_{x c}=E_{x c}[\phi, \epsilon] \\
\text { (with } \left.\phi=\left\{\phi_{1}, \ldots\right\}, \epsilon=\left\{\epsilon_{1}, \ldots\right\}\right)
\end{gathered}
$$

As the orbitals and eigenvalues themselves are (in general unknown) functionals of the density, $\phi_{k}=\phi_{k}[n], \epsilon_{k}=\epsilon_{k}[n]$, on arrives at 
the original statement of the HohenbergKohn (HK) theorem:

$$
E_{x c}=E_{x c}[\phi[n], \epsilon[n]]=E_{x c}[n]
$$

The natural definition for the exchange part of $E_{x c}$ :

$$
E_{x c}=E_{x}+E_{c}
$$

is the Fock term, expressed, however, in terms of KS orbitals ${ }^{46,47}$ :

$$
\begin{aligned}
E_{x}= & -\frac{e^{2}}{2} \int d^{3} r \int d^{3} r^{\prime} \\
& \times \sum_{\epsilon_{k}, \epsilon_{l} \leq \epsilon_{F}} \frac{\phi_{k}^{\dagger}(\mathbf{r}) \phi_{l}(\mathbf{r}) \phi_{l}^{\dagger}\left(\mathbf{r}^{\prime}\right) \phi_{k}\left(\mathbf{r}^{\prime}\right)}{\left|\mathbf{r}-\mathbf{r}^{\prime}\right|}
\end{aligned}
$$

This constitutes an implicit density functional that guarantees, in connection with the Hartree term, eq. (3), perfect cancelation of self-interaction effects. On the other hand, two questions immediately arise: (i) How can one generate: a multiplicative exchange potential, starting from eq. (8), rather than a nonlocal Hartree-Fock (HF)-type potential; and (ii) How can one generate a reasonable approximation for the correlation contribution, $E_{c}$, and the corresponding correlation potential $v_{c}$ ?

The first question and the last part of the second question are resolved by the optimized-potential-method (OPM) ${ }^{48,49}$ in which the multiplicative potential corresponding to any functional $E[\phi, \epsilon]$ can be determined via an integral equation. As the OPM selfconsistency procedure is much more involved than the direct KS scheme, this approach was initially applied only sparingly. In the meantime, however, an accurate approximation method for the solution of the crucial OPM integral equation has become available. ${ }^{50}$ The answer to the first part of the second question is still not fully available. The OPM has, for instance, been suggested ${ }^{51-54}$ for use with the self-interaction-corrected (SIC) $\mathrm{LDA}^{10}$ for exchange and correlation as a semiempirical orbital-dependent xc-functional. It is, however, not clear whether the SIC-LDA for correlation can serve as an adequate counterpart of the exact exchange. An additional semiempirical orbital-dependent ansatz for $E_{c}$, the functional due to Colle and Salvetti (CS) ${ }^{55}$ has also been put forward. ${ }^{56}$ The most promising ap- proach to $E_{c}$ is perturbation theory on the basis of the KS Hamiltonian ${ }^{57,58}$ (akin to Møller-Plesset perturbation theory on the basis of the HF Hamiltonian). A corresponding functional had initially been suggested by Görling and Levy. ${ }^{59}$

In what follows, we outline the basic formalism of the OPM, in the second section (an extension of the OPM to time-dependent systems is also available ${ }^{60}$ -we here, however, restrict ourselves to time-independent problems). This basic discussion is then complemented by an exposition of the salient features of the perturbative approach to the correlation problem (third section). In the fourth section, the method for the approximate solution due to Krieger, Li, and Iafrate (KLI) ${ }^{50}$ is described, comparing three variants for the treatment of eigenvalue-dependent functionals. Finally, we present some illustrative results for atoms ${ }^{45,49,61-64}$ as well as a brief overview of results for more complex systems. ${ }^{65-67}$

Before we proceed, we remark that the OPM can be extended to the relativistic domain, ${ }^{68-71}$ including the inverse electron-electron interaction and, at least in principle, all effects resulting from the creation of virtual electron-positron pairs. In this case, the basic DFT variable is the ground-state four current, the KS equations are of Dirac-type, and a set of four OPM integral equations determines the xc-four potential. Both the KLI approximation $^{70,71}$ and the perturbation expansion in terms of the relativistic KS Hamiltonian, ${ }^{71}$ for which quantum electrodynamics provides the appropriate framework, are also available in the relativistic situation. As in the nonrelativistic case, ${ }^{59}$ the perturbation approach allows the systematic derivation of an orbital-dependent correlation functional. ${ }^{71}$ However, a detailed discussion of the relativistic OPM and the various additional aspects involved, as the question of gauge invariance or the no-pair approximation, is beyond the scope of this study. For further details the interested reader is referred to ref. 71 .

\section{Optimized-Potential Method}

The HK theorem states that (under suitable mathematical conditions) the ground-state energy of a many-fermion system is a universal functional of the ground-state density. ${ }^{72}$ The guarantee of variational access, which accompanies the basic 
theorem, is usually exploited in the KS scheme where the density is represented in terms of single-particle orbitals. ${ }^{4}$ Two features make the KS approach a useful tool in many-body physics: (1) The fact that it represents (in principle) exact mapping of the interacting many-body problem onto an auxiliary single-particle problem, so that one can hope to go beyond the exchange-only limit. (2) The fact that the effective single particle potential, which itself is a functional of the density, is multiplicative (rather than an integral operator) and thus much easier to handle.

This efficiency is promoted by the fact that, in the conventional KS approach, the xc-potential is explicitly expressed in terms of only the density, eq. (4). On the other hand, in the OPM, the xc-energy is formulated as a functional of a set of KS orbitals, $\phi$, and KS eigenvalues, $\epsilon$, which themselves are unique functionals of the density via solution of an accompanying set of KS equations:

$$
\begin{gathered}
\left\{-\frac{\nabla^{2}}{2 m}+v_{s}(\mathbf{r})\right\} \phi_{k}(\mathbf{r})=\boldsymbol{\epsilon}_{k} \phi_{k}(\mathbf{r}) \\
v_{s}(\mathbf{r})=v_{e x t}(\mathbf{r})+v_{H}(\mathbf{r})+v_{x c}(\mathbf{r}) \\
v_{H}([n] ; \mathbf{r})=e^{2} \int d^{3} r^{\prime} \frac{n\left(\mathbf{r}^{\prime}\right)}{\left|\mathbf{r}-\mathbf{r}^{\prime}\right|} \\
v_{x c}([n] ; \mathbf{r})=\frac{\delta E_{x c}[n]}{\delta n(\mathbf{r})}
\end{gathered}
$$

For instance, the exchange part of $E_{x c}$ can then be taken to have the standard Fock form of eq. (8). The major task that arises if one bases the discussion on eq. (6) is the calculation of the multiplicative xc-potential, eq. (12), which is addressed by the OPM. ${ }^{48,49}$

Assume that an xc-functional of the form eq. (6) is given and that a set of solutions, $\phi_{k}(\mathbf{r})$ (with eigenvalues $\epsilon_{k}$ ), of the corresponding KS problem (both occupied and unoccupied levels) is available. The functional derivative, eq. (12), can then be evaluated with the chain rule for functional differentiation as:

$$
\begin{aligned}
v_{x c}(\mathbf{r})= & \int d^{3} \mathbf{r}^{\prime} \frac{\delta v_{s}\left(\mathbf{r}^{\prime}\right)}{\delta n(\mathbf{r})} \\
& \times \sum_{k}\left\{\int d^{3} r^{\prime \prime}\left[\frac{\delta \phi_{k}^{\dagger}\left(\mathbf{r}^{\prime \prime}\right)}{\delta v_{s}\left(\mathbf{r}^{\prime}\right)} \frac{\delta E_{x c}}{\delta \phi_{k}^{\dagger}\left(\mathbf{r}^{\prime \prime}\right)}+c . c .\right]\right. \\
& \left.+\frac{\delta \epsilon_{k}}{\delta v_{s}\left(\mathbf{r}^{\prime}\right)} \frac{\partial E_{x c}}{\partial \epsilon_{k}}\right\}
\end{aligned}
$$

The derivatives of the energy with respect to the orbitals and eigenvalues can be calculated once an explicit form for $E_{x c}$ is specified. In addition, one recognizes the density-density response function:

$$
\chi_{s}\left(\mathbf{r}, \mathbf{r}^{\prime}\right)=\frac{\delta n(\mathbf{r})}{\delta v_{s}\left(\mathbf{r}^{\prime}\right)}
$$

or, rather, its inverse:

$$
\chi_{s}^{-1}\left(\mathbf{r}, \mathbf{r}^{\prime}\right)=\frac{\delta v_{s}(\mathbf{r})}{\delta n\left(\mathbf{r}^{\prime}\right)}
$$

with

$$
\int d^{3} r^{\prime \prime} \chi_{s}^{-1}\left(\mathbf{r}, \mathbf{r}^{\prime \prime}\right) \chi_{s}\left(\mathbf{r}^{\prime \prime}, \mathbf{r}^{\prime}\right)=\delta^{(3)}\left(\mathbf{r}-\mathbf{r}^{\prime}\right)
$$

To obtain explicit expressions for the remaining factors in the integrand on the right-hand side of eq. (13), one considers the linear response of the KS system to a small perturbation $\delta v_{s}(\mathbf{r})$ :

$$
\left\{-\frac{\nabla^{2}}{2 m}+v_{s}(\mathbf{r})-\epsilon_{k}\right\} \delta \phi_{k}(\mathbf{r})=\left\{\delta \boldsymbol{\epsilon}_{k}-\delta v_{s}(\mathbf{r})\right\} \phi_{k}(\mathbf{r})
$$

which immediately yields:

$$
\begin{aligned}
\delta \boldsymbol{\epsilon}_{k} & =\int d^{3} r \phi_{k}^{\dagger}(\mathbf{r}) \phi_{k}(\mathbf{r}) \delta v_{s}(\mathbf{r}) \\
\delta \phi_{k}(\mathbf{r}) & =-\int d^{3} r^{\prime} G_{k}\left(\mathbf{r}, \mathbf{r}^{\prime}\right) \phi_{k}\left(\mathbf{r}^{\prime}\right) \delta v_{s}\left(\mathbf{r}^{\prime}\right) \\
G_{k}\left(\mathbf{r}, \mathbf{r}^{\prime}\right) & =\sum_{l \neq k} \frac{\phi_{l}(\mathbf{r}) \phi_{l}^{\dagger}\left(\mathbf{r}^{\prime}\right)}{\epsilon_{l}-\epsilon_{k}}
\end{aligned}
$$

One can directly show that the Green's function [eq. (20)] satisfies the differential equation:

$$
\begin{array}{r}
\left\{-\frac{\nabla^{2}}{2 m}+v_{s}(\mathbf{r})-\epsilon_{k}\right\} G_{k}\left(\mathbf{r}, \mathbf{r}^{\prime}\right) \\
=\delta^{(3)}\left(\mathbf{r}, \mathbf{r}^{\prime}\right)-\phi_{k}(\mathbf{r}) \phi_{k}^{\dagger}\left(\mathbf{r}^{\prime}\right)
\end{array}
$$

which will be used subsequently. One thus finds the relations:

$$
\begin{aligned}
& \frac{\delta \phi_{k}^{\dagger}(\mathbf{r})}{\delta v_{s}\left(\mathbf{r}^{\prime}\right)}=-\phi_{k}^{\dagger}\left(\mathbf{r}^{\prime}\right) G_{k}\left(\mathbf{r}^{\prime}, \mathbf{r}\right) ; \\
& \frac{\delta \boldsymbol{\epsilon}_{k}}{\delta v_{s}\left(\mathbf{r}^{\prime}\right)}=\phi_{k}^{\dagger}\left(\mathbf{r}^{\prime}\right) \phi_{k}\left(\mathbf{r}^{\prime}\right)
\end{aligned}
$$


Moreover, the response function [eq. (14)] can also be expressed in terms of the Green's function [eq. (20)]:

$$
\begin{aligned}
\chi_{s}\left(\mathbf{r}, \mathbf{r}^{\prime}\right)= & \sum_{k} \int d^{3} r^{\prime \prime}\left\{\frac{\delta n(\mathbf{r})}{\delta \phi_{k}\left(\mathbf{r}^{\prime \prime}\right)} \frac{\delta \phi_{k}\left(\mathbf{r}^{\prime \prime}\right)}{\delta v_{s}\left(\mathbf{r}^{\prime}\right)}\right. \\
& \left.+\frac{\delta n(\mathbf{r})}{\delta \phi_{k}^{\dagger}\left(\mathbf{r}^{\prime \prime}\right)} \frac{\delta \phi_{k}^{\dagger}\left(\mathbf{r}^{\prime \prime}\right)}{\delta v_{s}\left(\mathbf{r}^{\prime}\right)}\right\} \\
= & -\sum_{\epsilon_{k} \leq \epsilon_{F}} \phi_{k}^{\dagger}(\mathbf{r}) G_{k}\left(\mathbf{r}, \mathbf{r}^{\prime}\right) \phi_{k}\left(\mathbf{r}^{\prime}\right)+\text { c.c. }
\end{aligned}
$$

If one multiplies eq. (13) by $\chi_{s}\left(\mathbf{r}, \mathbf{r}^{\prime \prime \prime}\right)$ and integrates over $\mathbf{r}^{\prime \prime \prime}$ one obtains the OPM integral equation:

$$
\int d^{3} r^{\prime} \chi_{s}\left(\mathbf{r}, \mathbf{r}^{\prime}\right) v_{x c}\left(\mathbf{r}^{\prime}\right)=\Lambda_{x c}(\mathbf{r})
$$

where the kernel is given by eq. (23) and the right-hand side is:

$$
\begin{aligned}
\Lambda_{x c}(\mathbf{r})= & -\sum_{k} \int d^{3} r^{\prime}\left[\phi_{k}^{\dagger}(\mathbf{r}) G_{k}\left(\mathbf{r}, \mathbf{r}^{\prime}\right) \frac{\delta E_{x c}}{\delta \phi_{k}^{\dagger}\left(\mathbf{r}^{\prime}\right)}+c . c .\right] \\
& +\sum_{k} \phi_{k}^{\dagger}(\mathbf{r}) \phi_{k}(\mathbf{r}) \frac{\partial E_{x c}}{\partial \epsilon_{k}}
\end{aligned}
$$

All quantities involved are functionals of the KS orbitals and eigenvalues.

A necessary condition for the existence of a solution is obtained by integrating eq. (24) over $\mathbf{r}$ and using the projection property of $G_{k}\left(\mathbf{r}, \mathbf{r}^{\prime}\right)$ :

$$
\int d^{3} r \phi_{k}^{\dagger}(\mathbf{r}) G_{k}\left(\mathbf{r}, \mathbf{r}^{\prime}\right)=0
$$

which leads to:

$$
\sum_{k} \frac{\partial E_{x c}}{\partial \epsilon_{k}}=0
$$

Of course, this identity is satisfied by any first principles form of $E_{x c}$ [it is trivially satisfied by the exchange energy of eq. (8), but also the correlation functional derived in the next section complies with eq. (27)]. On the other hand, eq. (27) provides a consistency criterion for any semiempirical correlation functional.

The OPM integral equation also allows the verification of an important property of the exact exchange potential of finite systems ${ }^{49,58,73}$; that is, the fact that, asymptotically, the self-interaction potential of the outermost electron must be canceled by $v_{x}$ :

$$
v_{x}(\mathbf{r}) \underset{|\mathbf{r}| \rightarrow \infty}{\longrightarrow}-\frac{e^{2}}{|\mathbf{r}|}
$$

As a consequence, the exact exchange leads to a Rydberg series of unoccupied excited states, which is not present in the LDA or GGA. The OPM (using the exact $E_{x}$ ) is thus well suited to deal with negative ions. The discussion of the OPM integral equation in the asymptotic regime also allows one to establish an identity ${ }^{50}$ for the highest occupied KS level, $\phi_{h}$ :

$$
\int d^{3} r \phi_{h}^{\dagger}(\mathbf{r})\left\{\phi_{h}(\mathbf{r}) v_{x c}(\mathbf{r})-\frac{\delta E_{x c}}{\delta \phi_{h}^{\dagger}(\mathbf{r})}\right\}+\text { c.c. }=0
$$

Eq. (29) is particularly helpful for fixing the absolute normalization of $v_{x c}$, because, due to eq. (26), the OPM integral equation determines $v_{x c}$ only up to a global constant.

The technical problem one faces in the OPM is the combined self-consistency problem of solving the KS equations and the OPM integral equation simultaneously. This can be done directly with high numerical accuracy for spherical systems, ${ }^{25,45,49,61-64}$ which also allows for construction of OPM-based pseudopotentials. ${ }^{74}$ An algorithm suitable for condensed matter systems (within certain limitations, like the atomic-sphere approximation) has been suggested by Kotani. ${ }^{65}$ Recently, the OPM has also been formulated for a plane-wave basis, ${ }^{75}$ thus allowing plane-wave pseudopotential calculations for solids. ${ }^{67}$ Another candidate for an efficient algorithm is the KLI approximation (outlined in the fourth section).

\section{Perturbation Theory on Kohn-Sham Basis}

Although the exact $E_{x}$ has been introduced in the first section in a more intuitive way, we now present a systematic scheme for the derivation of orbital- and eigenvalue-dependent xc-functionals, taking up the basic idea of ref. 59. The approach rests on a perturbation expansion, using the KS system as a noninteracting reference system. ${ }^{57,58}$ Although, in practice, the total KS potential [eq. (10)] has to be calculated self-consistently, it is nevertheless legitimate to assume for the moment 
that $v_{s}$ is known. One must later face the task of disentangling the coupled evaluation of $E_{x c}$ and $v_{s}$. This potential then allows one to define a noninteracting KS Hamiltonian:

$$
\hat{H}_{s}=\int d^{3} x \hat{\psi}^{\dagger}(\mathbf{x})\left(-\frac{\nabla^{2}}{2 m}\right) \hat{\psi}(\mathbf{x})+\int d^{3} x \hat{n}(\mathbf{x}) v_{s}(\mathbf{x})
$$

The KS problem is assumed to be solved; that is, in particular, the noninteracting many-particle ground state $\left|\Phi_{s}\right\rangle$ and the eigenvalue $E_{s}$ are known:

$$
\hat{H}_{s}\left|\Phi_{s}\right\rangle=E_{s}\left|\Phi_{s}\right\rangle ; \quad E_{s}=T_{s}+\int d^{3} \times n(\mathbf{x}) v_{s}(\mathbf{x})
$$

To obtain a formula for the energy difference between the exact ground-state energy, E:

$$
\hat{H}|\Phi\rangle=E|\Phi\rangle
$$

and the noninteracting energy, $E_{s}$, and thus for $E_{x c}$, we use a perturbation expansion in terms of $\hat{H}-\hat{H}_{s}$. Low-order contributions of this expansion could be obtained on the basis of the standard Rayleigh-Schrödinger perturbation theory. However, for the sake of providing a more general result for $E_{x c}$ we rely here on a coupling constant integration technique. The interaction Hamiltonian is thus augmented by a dimensionless coupling strength parameter, $g$ :

$$
\begin{gathered}
\hat{H}(g)=\hat{H}_{s}+g \hat{H}_{1} \\
\hat{H}_{1}=\frac{e^{2}}{2} \int d^{3} x \int d^{3} y \frac{\hat{\psi}^{\dagger}(\mathbf{x}) \hat{\psi}^{\dagger}(\mathbf{y}) \hat{\psi}(\mathbf{y}) \hat{\psi}(\mathbf{x})}{|\mathbf{x}-\mathbf{y}|} \\
-\int d^{3} x v_{H x c}(\mathbf{x}) \hat{n}(\mathbf{x}) \\
v_{H x c}(\mathbf{x}) \equiv v_{s}(\mathbf{x})-v_{e x t}(\mathbf{x})=v_{H}(\mathbf{x})+v_{x c}(\mathbf{x})
\end{gathered}
$$

The original Hamiltonian is obtained from eq. (33) for $g=1$. The desired expression for the energy shift induced by the interaction is obtained by first differentiating the $g$-dependent ground state $E(g)$ $=\langle\Phi(g)|\hat{H}(g)| \Phi(g)\rangle$ with respect to the coupling parameter, and subsequently integrating over $g$ :

$$
\begin{aligned}
E(1)-E(0) & =E-E_{s} \equiv E_{1} \\
& =\int_{0}^{1} d g\left\langle\Phi(g)\left|\hat{H}_{1}\right| \Phi(g)\right\rangle
\end{aligned}
$$

To obtain a more explicit result for $E_{x c}$ one can now utilize the concept of adiabatic switching ${ }^{76}$ for $\hat{H}_{1}$. Assuming the interacting ground state, $|\Phi(g)\rangle$, emerges from the noninteracting ground state, $\left|\Phi_{s}\right\rangle$, as one increases $g$ from 0 to 1 and both states to be nondegenerate, the connection between them is established via the interaction-picture time-evolution operator $\hat{U}_{I, \epsilon}$ :

$$
\begin{gathered}
|\Phi\rangle=A \lim _{\epsilon \rightarrow 0} \frac{\hat{U}_{I, \epsilon}(0, \mp \infty)\left|\Phi_{s}\right\rangle}{\left\langle\Phi_{s}\left|\hat{U}_{I, \epsilon}(0, \mp \infty)\right| \Phi_{s}\right\rangle} \\
A=\lim _{\epsilon_{1}, \epsilon_{2} \rightarrow 0}\left[\frac{\left\langle\Phi_{s}\left|\hat{U}_{I, \epsilon_{1}}(+\infty, 0)\right| \Phi_{s}\right\rangle\left\langle\Phi_{s}\left|\hat{U}_{I, \epsilon_{2}}(0,-\infty)\right| \Phi_{s}\right\rangle}{\left\langle\Phi_{s}\left|\hat{U}_{I, \epsilon_{1}}(+\infty, 0) \hat{U}_{I, \epsilon_{2}}(0,-\infty)\right| \Phi_{s}\right\rangle}\right]_{(38)}^{1 / 2} \\
\hat{U}_{I, \epsilon}\left(t, t^{\prime}\right)=\sum_{n=0}^{\infty} \frac{(-i g)^{n}}{n !} \\
\quad \times \int_{t^{\prime}}^{t} d t_{1} \cdots \int_{t^{\prime}}^{t} d t_{n} e^{-\epsilon\left(\left|t_{1}\right|+\cdots+\left|t_{n}\right|\right)} \\
\quad \times T\left[\hat{H}_{1, I}\left(t_{1}\right) \cdots \hat{H}_{1, I}\left(t_{n}\right)\right] \\
\hat{H}_{1, I}(t)=e^{i \hat{H}_{s} t} \hat{H}_{1} e^{-i \hat{H}_{s} t} \\
=\frac{e^{2}}{2} \int d^{3} x \int d^{3} y \\
\hat{n}_{I}(\mathbf{x}, t)=e^{i \hat{H}_{s} t} \hat{n}(\mathbf{x}) e^{-i \hat{H}_{s} t} \\
\hat{\psi}_{I}(\mathbf{x}, t)=e^{i \hat{H}_{s} t} \hat{\psi}(\mathbf{x}) e^{-i \hat{H}_{s} t} \\
\quad \times \frac{\hat{\psi}_{I}^{\dagger}(\mathbf{x}, t) \hat{\psi}_{I}^{\dagger}(\mathbf{y}, t) \hat{\psi}_{I}(\mathbf{y}, t) \hat{\psi}_{I}(\mathbf{x}, t)}{|\mathbf{x}-\mathbf{y}|} \\
-\int d^{3} x v_{H x c}(\mathbf{x}) \hat{n}_{I}(\mathbf{x}, t)
\end{gathered}
$$

(where $A$ ensures the $g$-independent normalization of $|\Phi(g)\rangle)$. Insertion of eq. (37) into eq. (36), and use of the additivity of the time-evolution operator, gives:

$$
\begin{aligned}
E_{1}= & \lim _{\epsilon \rightarrow 0} \int_{0}^{1} d g \sum_{n=0}^{\infty} \frac{(-i g)^{n}}{n !} \int_{-\infty}^{\infty} d t_{1} \cdots \\
& \int_{-\infty}^{\infty} d t_{n} e^{-\epsilon\left(\left|t_{1}\right|+\cdots+\left|t_{n}\right|\right)} \\
& \times \frac{\left\langle\Phi_{s}\left|T \hat{H}_{1, I}(0) \hat{H}_{1, I}\left(t_{1}\right) \cdots \hat{H}_{1, I}\left(t_{n}\right)\right| \Phi_{s}\right\rangle}{\left\langle\Phi_{s}\left|\hat{U}_{I, \epsilon}(+\infty,-\infty)\right| \Phi_{s}\right\rangle}
\end{aligned}
$$


The denominator in eq. (41) exactly cancels those contributions to a diagrammatic expansion of the numerator which contain at least one subdiagram not connected to the vertex defined by $\hat{H}_{1, I}(0)$. Restricting the diagrammatic evaluation of the numerator to connected diagrams, the coupling constant integration can be performed. Extracting the $x_{C}$ contribution from $E_{1}$ via eqs. (1), (3), (33), (34), and (36) the final result for $E_{x c}$ can be written as:

$$
\begin{aligned}
E_{x c}= & \frac{e^{2}}{2} \int d^{3} x \int d^{3} y \\
& \times \frac{\left\langle\Phi_{s}\left|\hat{\psi}^{\dagger}(\mathbf{x}) \hat{\psi}^{\dagger}(\mathbf{y}) \hat{\psi}(\mathbf{y}) \hat{\psi}(\mathbf{x})\right| \Phi_{s}\right\rangle-n(\mathbf{x}) n(\mathbf{y})}{|\mathbf{x}-\mathbf{y}|} \\
& +\sum_{n=1}^{\infty} \frac{(-i)^{n}}{(n+1) !} \int_{-\infty}^{\infty} d t_{1} \cdots \int_{-\infty}^{\infty} d t_{n} \\
& \times\left\langle\Phi_{s}\left|T \hat{H}_{1, I}(0) \hat{H}_{1, I}\left(t_{1}\right) \cdots \hat{H}_{1, I}\left(t_{n}\right)\right| \Phi_{s}\right\rangle_{c}
\end{aligned}
$$

where the index $c$ indicates that only connected diagrams have to be taken into account. ${ }^{77} \mathrm{Eq}$. (42) provides an exact representation of $E_{x c}$ in terms of the KS orbitals, the KS eigenvalues, and the xcpotential; that is, the functional derivative of $E_{x c}$ with respect to $n$. Thus, eq. (42) is not directly suitable for practical calculations, as a consequence of the fact that we have used a noninteracting reference Hamiltonian that is determined self-consistently. However, this intricate coupling pattern can be resolved by an expansion in powers of $e^{2}$ :

$$
\begin{gathered}
E_{x c}=E_{x}+\sum_{i=2}^{\infty} E_{c}^{(i)} ; \quad v_{x c}=v_{x}+\sum_{i=2}^{\infty} v_{c}^{(i)} ; \\
E_{x}, v_{x} \sim e^{2} ; \quad E_{c}^{(i)}, v_{c}^{(i)} \sim e^{2 i}
\end{gathered}
$$

where the lowest order contribution, the exchange energy, has been extracted explicitly. After insertion of eq. (45) into eq. (42), one obtains $E_{x}$ in the form eq. (8), as expected. The corresponding exchange potential can thus be evaluated using the OPM as discussed previously:

$$
\begin{gathered}
\int d^{3} r^{\prime} \chi_{s}\left(\mathbf{r}, \mathbf{r}^{\prime}\right) v_{x}\left(\mathbf{r}^{\prime}\right)=\Lambda_{x}(\mathbf{r}) \\
\Lambda_{x}(\mathbf{r})=-\sum_{k} \int d^{3} r^{\prime}\left[\phi_{k}^{\dagger}(\mathbf{r}) G_{k}\left(\mathbf{r}, \mathbf{r}^{\prime}\right) \frac{\delta E_{x}}{\delta \phi_{k}^{\dagger}\left(\mathbf{r}^{\prime}\right)}+\text { c.c. }\right]
\end{gathered}
$$

For the lowest order correlation contribution one finds:

$$
\begin{aligned}
E_{c}^{(2)}= & \frac{e^{4}}{2} \sum_{\epsilon_{i}, \epsilon_{j} \leq \epsilon_{F}<\epsilon_{k}, \epsilon_{l}} \frac{1}{\epsilon_{i}+\epsilon_{j}-\epsilon_{k}-\epsilon_{l}} \\
& \times \int d^{3} r_{1} \int d^{3} r_{2} \frac{\phi_{i}^{\dagger}\left(\mathbf{r}_{1}\right) \phi_{k}\left(\mathbf{r}_{1}\right) \phi_{j}^{\dagger}\left(\mathbf{r}_{2}\right) \phi_{l}\left(\mathbf{r}_{2}\right)}{\left|\mathbf{r}_{1}-\mathbf{r}_{2}\right|} \\
& \times \int d^{3} r_{3} \int d^{3} r_{4}\left\{\frac{\phi_{k}^{\dagger}\left(\mathbf{r}_{3}\right) \phi_{i}\left(\mathbf{r}_{3}\right) \phi_{l}^{\dagger}\left(\mathbf{r}_{4}\right) \phi_{j}\left(\mathbf{r}_{4}\right)}{\left|\mathbf{r}_{3}-\mathbf{r}_{4}\right|}\right. \\
& \left.-\frac{\phi_{k}^{\dagger}\left(\mathbf{r}_{3}\right) \phi_{j}\left(\mathbf{r}_{3}\right) \phi_{l}^{\dagger}\left(\mathbf{r}_{4}\right) \phi_{i}\left(\mathbf{r}_{4}\right)}{\left|\mathbf{r}_{3}-\mathbf{r}_{4}\right|}\right\} \\
& +\sum_{\epsilon_{k} \leq \epsilon_{F}<\epsilon_{l}} \frac{1}{\epsilon_{k}-\epsilon_{l}} \\
& \times \mid \int d^{3} r_{1} \phi_{k}^{\dagger}\left(\mathbf{r}_{1}\right) \phi_{l}\left(\mathbf{r}_{1}\right) v_{x}\left(\mathbf{r}_{1}\right)+e^{2} \sum \\
& \times \int d^{3} r_{1} \int d^{3} r_{2} \frac{\phi_{k}^{\dagger}\left(\mathbf{r}_{1}\right) \phi_{j}\left(\mathbf{r}_{1}\right) \phi_{j}^{\dagger}\left(\mathbf{r}_{2}\right) \phi_{l}\left(\mathbf{r}_{2}\right)}{\left|\mathbf{r}_{1}-\mathbf{r}_{2}\right|}
\end{aligned}
$$

Due to the fact that $v_{x}$ can be calculated via eqs. (44) and (45) (i.e., $v_{x}$ is itself a functional of the $\phi_{k}$ and $\left.\epsilon_{k}\right), E_{c}^{(2)}$ is a well-defined functional. The corresponding potential, $v_{c}^{(2)}$, can again be evaluated by the OPM:

$$
\int d^{3} r_{2} \chi_{s}\left(\mathbf{r}_{1}, \mathbf{r}_{2}\right) v_{c}^{(2)}\left(\mathbf{r}_{2}\right)=\Lambda_{c}^{(2)}\left(\mathbf{r}_{1}\right)
$$

taking into account the $v_{x}$-dependence of $E_{c}^{(2)}$ :

$$
\begin{aligned}
\Lambda_{c}^{(2)}\left(\mathbf{r}_{1}\right)= & -\sum_{k} \int d^{3} r_{2}\left[\phi_{k}^{\dagger}\left(\mathbf{r}_{1}\right) G_{k}\left(\mathbf{r}_{1}, \mathbf{r}_{2}\right)\right. \\
& \left.\times \frac{\delta E_{c}^{(2)}}{\delta \phi_{k}^{\dagger}\left(\mathbf{r}_{2}\right)}+c . c .\right] \\
& +\sum_{k} \phi_{k}^{\dagger}\left(\mathbf{r}_{1}\right) \phi_{k}\left(\mathbf{r}_{1}\right) \frac{\partial E_{c}^{(2)}}{\partial \epsilon_{k}} \\
& +\int d^{3} r_{2} \frac{\delta v_{x}\left(\mathbf{r}_{2}\right)}{\delta v_{s}\left(\mathbf{r}_{1}\right)} \frac{\delta E_{c}^{(2)}}{\delta v_{x}\left(\mathbf{r}_{2}\right)},
\end{aligned}
$$

where the last term has been simplified via:

$$
\int d^{3} r_{3} \chi_{s}\left(\mathbf{r}_{3}, \mathbf{r}_{1}\right) \frac{\delta v_{x}\left(\mathbf{r}_{2}\right)}{\delta n\left(\mathbf{r}_{3}\right)}=\frac{\delta v_{x}\left(\mathbf{r}_{2}\right)}{\delta v_{s}\left(\mathbf{r}_{1}\right)}
$$


The functional derivatives of $E_{x}$ and $E_{c}^{(2)}$ with respect to $\phi_{k}, \epsilon_{k}$, and $v_{x}$ can be performed directly, using their explicit forms [eqs. (8) and (46)]. It remains to calculate $\delta v_{x} / \delta v_{s}$. Taking the functional derivative of the $x$-only OPM equation, eq. (44), with respect to $v_{s}$, one finds, after reordering:

$$
\begin{aligned}
\frac{\delta v_{x}\left(\mathbf{r}_{2}\right)}{\delta v_{s}\left(\mathbf{r}_{1}\right)}= & \int d^{3} r_{3} \chi_{s}^{-1}\left(\mathbf{r}_{2}, \mathbf{r}_{3}\right)\left\{\frac{\delta \Lambda_{x}\left(\mathbf{r}_{3}\right)}{\delta v_{s}\left(\mathbf{r}_{1}\right)}\right. \\
& \left.-\int d^{3} r_{4} \frac{\delta \chi_{s}\left(\mathbf{r}_{3}, \mathbf{r}_{4}\right)}{\delta v_{s}\left(\mathbf{r}_{1}\right)} v_{x}\left(\mathbf{r}_{4}\right)\right\}
\end{aligned}
$$

The first unknown ingredient of eq. (49) can be evaluated using the standard OPM replacement of derivatives with respect to $v_{s}$ by derivatives with respect to the orbitals and eigenvalues:

$$
\begin{aligned}
\frac{\delta \Lambda_{x}\left(\mathbf{r}_{2}\right)}{\delta v_{s}\left(\mathbf{r}_{1}\right)}= & -\sum_{k} \int d^{3} r_{3} \\
& \times\left[\phi_{k}^{\dagger}\left(\mathbf{r}_{1}\right) G_{k}\left(\mathbf{r}_{1}, \mathbf{r}_{3}\right) \frac{\delta \Lambda_{x}\left(\mathbf{r}_{2}\right)}{\delta \phi_{k}^{\dagger}\left(\mathbf{r}_{3}\right)}+c . c .\right] \\
& +\sum_{k} \phi_{k}^{\dagger}\left(\mathbf{r}_{1}\right) \phi_{k}\left(\mathbf{r}_{1}\right) \frac{\partial \Lambda_{x}\left(\mathbf{r}_{2}\right)}{\partial \epsilon_{k}}
\end{aligned}
$$

The second contribution, eq. (49), requires the evaluation of the quadratic response function:

$$
\begin{aligned}
\frac{\delta \chi_{s}\left(\mathbf{r}_{1}, \mathbf{r}_{2}\right)}{\delta v_{s}\left(\mathbf{r}_{3}\right)}= & \frac{\delta^{2} n\left(\mathbf{r}_{1}\right)}{\delta v_{s}\left(\mathbf{r}_{3}\right) \delta v_{s}\left(\mathbf{r}_{2}\right)} \\
= & \sum_{\epsilon_{k} \leq \epsilon_{F}} H_{k}\left(\mathbf{r}_{1}, \mathbf{r}_{2}, \mathbf{r}_{3}\right) \\
& + \text { all permutations of } \mathbf{r}_{1} \leftrightarrow \mathbf{r}_{2} \leftrightarrow \mathbf{r}_{3},
\end{aligned}
$$

with:

$$
\begin{aligned}
H_{k}\left(\mathbf{r}_{1}, \mathbf{r}_{2}, \mathbf{r}_{3}\right)= & \phi_{k}^{\dagger}\left(\mathbf{r}_{1}\right) G_{k}\left(\mathbf{r}_{1}, \mathbf{r}_{2}\right) G_{k}\left(\mathbf{r}_{2}, \mathbf{r}_{3}\right) \phi_{k}\left(\mathbf{r}_{3}\right) \\
& -\phi_{k}^{\dagger}\left(\mathbf{r}_{1}\right) \phi_{k}\left(\mathbf{r}_{1}\right) \phi_{k}^{\dagger}\left(\mathbf{r}_{2}\right) \int d^{3} r_{4} \\
& \times G_{k}\left(\mathbf{r}_{2}, \mathbf{r}_{4}\right) G_{k}\left(\mathbf{r}_{4}, \mathbf{r}_{3}\right) \phi_{k}\left(\mathbf{r}_{3}\right)
\end{aligned}
$$

Eqs. (8), (46)-(52) completely determine $v_{c}^{(2)}$.

The generalization of this procedure to higher orders is now obvious. The correlation energy, $E_{c}^{(n)}$, of order $e^{2 n}$ depends only on the correlation potentials of lower order. $E_{c}^{(n)}$ is thus completely defined, once the lower order potentials have been evaluated via the corresponding OPM integral equations. Finally, using the OPM also for the order $e^{2 n}$ allows one to calculate $v_{c}^{(n)}$. This establishes a recursive definition of $E_{c}$ in terms of the $\phi_{k}^{(\dagger)}$ and $\epsilon_{k}$.

In practice, however, a rigorous application of this iteratively defined $E_{c}$ would require the evaluation of response functions of arbitrary order. Thus, while eq. (42) is exact in principle, some approximate handling of the $v_{x c}$-dependence in eq. (42) seems unavoidable, if one wants to go beyond the lowest order scheme discussed here. It remains to be investigated whether, for instance, an LDA treatment for the crucial ingredient of the OPM equation, the xc-kernel $f_{x c}=\delta v_{x c} / \delta n$, is sufficient for this purpose.

It is worthwhile to analyze the energy [eq. (46)] in slightly more detail. First, one recognizes that it consists of two contributions, the first of which has exactly the same structure as the standard secondorder Møller-Plesset (MP2) correlation energy, resulting from use of the HF Hamiltonian, $\hat{H}_{H F}$, as the noninteracting reference system. Correspondingly, the second term represents the difference between the x-only OPM and HF ground-state energies, evaluated to lowest order in $\hat{H}_{H F}-\hat{H}_{s}$. As is well known, this difference is rather small ${ }^{64}$ (see later), suggesting that the MP2-like contribution to $E_{c}^{(2)}$ alone might be a useful approximation for the correlation energy functional. ${ }^{71}$

In view of the close analogy of $E_{c}^{(2)}$ with the MP2 correlation energy, it is not surprising that $E_{c}^{(2)}$ includes long-range dispersive (van der Waals) forces. ${ }^{71}$ This property is most easily verified for two neutral atoms, A and B, whose centers are separated by a large distance, $R$, so that the KS orbitals are localized around the two atomic centers and the orbitals corresponding to atom A have no overlap with those centered on atom $B$ (i.e., the overlap vanishes exponentially with $R$ ). Each sum over the KS levels in eq. (46) can then be split into two sums over orbitals belonging to the centers A and B:

$$
\sum_{\epsilon_{i}} \rightarrow \sum_{\epsilon_{i_{A}}}+\sum_{\epsilon_{i_{B}}}
$$

All spatial integrals in eq. (46) that combine orbitals from both centers, $\phi_{i_{A}}^{\dagger}(\mathbf{r}) \phi_{k_{B}}(\mathbf{r})$, vanish exponentially for large $R$. As a consequence, $E_{c}^{(2)}$ can be decomposed into the corresponding correlation energies of the two atoms and an interaction contri- 
bution:

$$
\begin{aligned}
E_{c, \text { int }}^{(2)}= & \sum_{c}^{(2)} \underset{\epsilon_{i_{A}} \leq \epsilon_{F}<\epsilon_{k_{A}}}{\underset{\epsilon_{i_{B}} \leq \epsilon_{F}<\epsilon_{l_{B}}}{\longrightarrow}} E_{c, A}^{(2)}+E_{c, B}^{(2)}+E_{c, i n t}^{(2)} \\
& \times \mid \int d^{3} r_{1} \int d^{3} r_{2} \\
& \times\left.\frac{\phi_{i_{A}}^{\dagger}\left(\mathbf{r}_{1}\right) \phi_{j_{B}}-\epsilon_{k_{A}}-\epsilon_{l_{B}}}{\left.\mid \mathbf{r}_{1}\right) \phi_{j_{B}}^{\dagger}\left(\mathbf{r}_{2}\right) \phi_{l_{B}}\left(\mathbf{r}_{2}\right)}\right|^{2}
\end{aligned}
$$

Whereas $E_{c, A}^{(2)}$ and $E_{c, B}^{(2)}$ have the same structure as eq. (46), the interaction term, $E_{c, i n t}^{(2)}$, originates from the direct contribution to the MP2-like term in eq. (46). Introducing the time-ordered KS response functions:

$$
\begin{aligned}
\chi_{s}\left(\mathbf{r}_{1}, \mathbf{r}_{2}, \omega\right)= & \sum_{\epsilon_{k} \leq \epsilon_{F}<\epsilon_{l}}\left\{\frac{\phi_{k}^{\dagger}\left(\mathbf{r}_{1}\right) \phi_{l}\left(\mathbf{r}_{1}\right) \phi_{l}^{\dagger}\left(\mathbf{r}_{2}\right) \phi_{k}\left(\mathbf{r}_{2}\right)}{\omega+\epsilon_{k}-\epsilon_{l}+i \eta}\right. \\
& \left.-\frac{\phi_{l}^{\dagger}\left(\mathbf{r}_{1}\right) \phi_{k}\left(\mathbf{r}_{1}\right) \phi_{k}^{\dagger}\left(\mathbf{r}_{2}\right) \phi_{l}\left(\mathbf{r}_{2}\right)}{\omega-\epsilon_{k}+\epsilon_{l}-i \eta}\right\}
\end{aligned}
$$

of the individual atoms, one can rewrite eq. (54) as:

$$
\begin{aligned}
E_{c, \text { int }}^{(2)}= & -\frac{e^{4}}{2} \int d^{3} r_{1} \cdots d^{3} r_{4} \\
& \times \int \frac{d \omega}{2 \pi i} \frac{\chi_{s, A}\left(\mathbf{r}_{1}, \mathbf{r}_{3}, \omega\right) \chi_{s, B}\left(\mathbf{r}_{4}, \mathbf{r}_{2}, \omega\right)}{\left|\mathbf{r}_{1}-\mathbf{r}_{2}\right|\left|\mathbf{r}_{3}-\mathbf{r}_{4}\right|}
\end{aligned}
$$

Eq. (56) does not yet have the familiar form ${ }^{78}$ of the van der Waals interaction. However, using time-reversal invariance, the time-ordered response function can be replaced by the retarded $\mathrm{KS}$ response function:

$$
\begin{gathered}
\chi_{s}^{R}\left(\mathbf{r}_{1}, \mathbf{r}_{2}, \omega\right) \\
=\sum_{\epsilon_{k} \leq \epsilon_{F}<\epsilon_{l}}\left\{\frac{\phi_{k}^{\dagger}\left(\mathbf{r}_{1}\right) \phi_{l}\left(\mathbf{r}_{1}\right) \phi_{l}^{\dagger}\left(\mathbf{r}_{2}\right) \phi_{k}\left(\mathbf{r}_{2}\right)}{\omega+\epsilon_{k}-\epsilon_{l}+i \eta}\right. \\
\left.-\frac{\phi_{l}^{\dagger}\left(\mathbf{r}_{1}\right) \phi_{k}\left(\mathbf{r}_{1}\right) \phi_{k}^{\dagger}\left(\mathbf{r}_{2}\right) \phi_{l}\left(\mathbf{r}_{2}\right)}{\omega-\epsilon_{k}+\epsilon_{l}+i \eta}\right\} \\
\chi_{s}^{R}\left(\mathbf{r}_{1}, \mathbf{r}_{2}, \omega\right)= \begin{cases}\chi_{s}\left(\mathbf{r}_{1}, \mathbf{r}_{2}, \omega\right) & \text { for } \omega \geq 0 \\
\chi_{s}\left(\mathbf{r}_{1}, \mathbf{r}_{2}, \omega\right)^{*} & \text { for } \omega \leq 0\end{cases}
\end{gathered}
$$

Finally, choosing the center of atom $\mathrm{A}$ as origin for the $\mathbf{r}_{1}$ and $\mathbf{r}_{3}$ integrations and the center of atom $B$ as origin for the $\mathbf{r}_{2}$ and $\mathbf{r}_{4}$ integrations, one can easily expand the Coulomb interaction for this choice of coordinates,

$$
\begin{aligned}
\frac{1}{\left|\mathbf{r}_{1}-\mathbf{r}_{2}\right|} \rightarrow & \frac{1}{\left|\mathbf{R}+\mathbf{r}_{1}-\mathbf{r}_{2}\right|} \\
= & \frac{1}{R}\left\{1-\frac{\left(\mathbf{r}_{1}-\mathbf{r}_{2}\right) \cdot \mathbf{R}}{R^{2}}\right. \\
& \left.+\frac{3\left[\left(\mathbf{r}_{1}-\mathbf{r}_{2}\right) \cdot \mathbf{R}\right]^{2}}{2 R^{4}}-\frac{\left(\mathbf{r}_{1}-\mathbf{r}_{2}\right)^{2}}{2 R^{2}}+\cdots\right\}
\end{aligned}
$$

The two leading terms resulting from insertion of this expansion into eq. (56) do not contribute due to particle number conservation, so that one ends up with:

$$
\begin{aligned}
E_{c, i n t}^{(2)}= & -\frac{e^{4}}{R^{6}} \int_{0}^{\infty} \frac{d u}{2 \pi} \sum_{i j k l}\left(\delta_{i j}-3 \frac{R^{i} R^{j}}{R^{2}}\right) \\
& \times\left(\delta_{k l}-3 \frac{R^{k} R^{l}}{R^{2}}\right) \alpha_{A, i k}(i u) \alpha_{B, j l}(i u)
\end{aligned}
$$

where $\alpha_{i k}$ is the atomic KS polarizability tensor:

$$
\alpha_{i k}(\omega)=\int d^{3} r_{1} \int d^{3} r_{2} r_{1}^{i} r_{2}^{k} \chi_{s}^{R}\left(\mathbf{r}_{1}, \mathbf{r}_{2}, \omega\right)
$$

Although the KS polarizability is not identical to the full atomic polarizability, $E_{c}^{(2)}$ nevertheless reproduces van der Waals interaction between two atoms, at least qualitatively (in contrast to the LDA and the GGA). Moreover, a first evaluation of the polarizabilities, eq. (60), ${ }^{79}$ shows that, for light atoms, eq. (59) provides a reasonable approximation for the lowest order van der Walls coefficient, $\mathrm{C}_{6}$.

\section{Krieger-Li-Iafrate Approximation}

The numerical solution of the integral equation [eq. (24)] is computationally rather demanding, so that an approximate analytical or at least semianalytical solution substantially increases the range of applicability of the OPM. Such a scheme has been suggested by Krieger, Li, and Iafrate (KLI). ${ }^{50}$ KLI have pointed out two different routes for the derivation of their approximation to the OPM integral equation: One can either rely on a closure approximation for the Green's function [eq. (28) $]^{50}$ 
or rewrite the OPM integral equation in a form that suggests the neglect of certain complicated "higher order" contributions. ${ }^{62}$ A third derivation, although being in the spirit of the second scheme, has been given in ref. 70. All three approaches have originally been formulated for purely orbitaldependent $E_{x c}$, like the exchange-only energy [eq. (8)]. However, $E_{x c}$ may also depend on the KS eigenvalues $\epsilon_{k}$ (an explicit example was given earlier). This situation, for which the three approaches to the KLI approximation lead to different results for $v_{x c}^{K L I}, 71$ was first analyzed in the context of the relativistic exchange energy, which also is eigenvalue-dependent, due to the transverse electron-electron interaction.

The KLI approximation is most easily introduced via the closure approach: Replacing the denominator in eq. (20) by some average eigenvalue difference, $\Delta \bar{\epsilon}$, one obtains:

$$
\begin{aligned}
G_{k}\left(\mathbf{r}, \mathbf{r}^{\prime}\right) & \approx \sum_{l \neq k} \frac{\phi_{l}(\mathbf{r}) \phi_{l}^{\dagger}\left(\mathbf{r}^{\prime}\right)}{\Delta \bar{\epsilon}} \\
& =\frac{\delta^{(3)}\left(\mathbf{r}-\mathbf{r}^{\prime}\right)-\phi_{k}(\mathbf{r}) \phi_{k}^{\dagger}\left(\mathbf{r}^{\prime}\right)}{\Delta \overline{\boldsymbol{\epsilon}}}
\end{aligned}
$$

Insertion into eq. (24) leads directly to the first form (a) of the KLI approximation for $v_{x c}$ :

$$
\begin{aligned}
v_{x c}^{K L I a}(\mathbf{r})= & \frac{1}{2 n(\mathbf{r})} \\
& \times\left\{\sum_{k}\left[\phi_{k}^{\dagger}(\mathbf{r}) \frac{\delta E_{x c}}{\delta \phi_{k}^{\dagger}(\mathbf{r})}-n_{k}(\mathbf{r}) \bar{e}_{k}+c . c .\right]\right. \\
& \left.+2 \sum_{\epsilon_{k} \leq \epsilon_{F}} n_{k}(\mathbf{r}) \bar{v}_{k}-\Delta \overline{\boldsymbol{\epsilon}} \sum_{k} n_{k}(\mathbf{r}) \frac{\partial E_{x c}}{\partial \boldsymbol{\epsilon}_{k}}\right\}
\end{aligned}
$$

with:

$$
\begin{aligned}
\bar{e}_{k} & =\int d^{3} r \phi_{k}^{\dagger}(\mathbf{r}) \frac{\delta E_{x c}}{\delta \phi_{k}^{\dagger}(\mathbf{r})} \\
\bar{v}_{k} & =\int d^{3} r n_{k}(\mathbf{r}) v_{x c}(\mathbf{r}) \\
n_{k}(\mathbf{r}) & =\phi_{k}^{\dagger}(\mathbf{r}) \phi_{k}(\mathbf{r}) .
\end{aligned}
$$

Eq. (62) is still an integral equation for the unknown potential, $v_{x c}$, due to the $v_{x c}$-dependence of the right-hand side via $\bar{v}_{k}$. The coefficients, $\bar{v}_{k}$, however, can be determined by multiplication of eq. (62) with $n_{l}(\mathbf{r})$ and subsequent integration over r:

$$
\begin{aligned}
\bar{v}_{l}= & \int d^{3} r \frac{n_{l}(\mathbf{r})}{2 n(\mathbf{r})} \\
& \times\left\{\sum_{k}\left[\phi_{k}^{\dagger}(\mathbf{r}) \frac{\delta E_{x c}}{\delta \phi_{k}^{\dagger}(\mathbf{r})}-n_{k}(\mathbf{r}) \bar{e}_{k}+c . c .\right]\right. \\
& \left.+2 \sum_{\epsilon_{k} \leq \epsilon_{F}} n_{k}(\mathbf{r}) \bar{v}_{k}-\Delta \bar{\epsilon} \sum_{k} n_{k}(\mathbf{r}) \frac{\partial E_{x c}}{\partial \epsilon_{k}}\right\}
\end{aligned}
$$

Eq. (66) represents a set of linear equations for the unknown coefficients, $\bar{v}_{k}$, which can be solved for given $\phi_{k}, \epsilon_{k}$, and $\Delta \bar{\epsilon}$, but without prior knowledge of $v_{x c}$. This then reduces eq. (62) to a simple analytical relation.

Eq. (62) explicitly illustrates the ambiguity of the KLI approximation for eigenvalue-dependent functions just mentioned: Whereas for the exchange energy [eq. (8)] and the semiempirical Colle-Salvetti functional ${ }^{55}$ the $\Delta \bar{\epsilon}$-dependent term on the right-hand side vanishes, any eigenvaluedependent $E_{x c}$ requires an explicit specification of $\Delta \bar{\epsilon}$. Because in this way a characteristic energy scale of the system is introduced by hand, the KLI approximation loses its universality.

One would hope that the alternative approaches to the KLI approximation resolve this problem as they do not rely on approximation like eq. (61). In these schemes, the discussion is based on an exact reformulation of the OPM integral equation, which can be written as:

$$
\begin{aligned}
& {\left[\sum_{k} \boldsymbol{\phi}_{k}^{\dagger}(\mathbf{r}) \int d^{3} r^{\prime} G_{k}\left(\mathbf{r}, \mathbf{r}^{\prime}\right)\right.} \\
& \left.\quad \times\left(\phi_{k}\left(\mathbf{r}^{\prime}\right) v_{x c}\left(\mathbf{r}^{\prime}\right)-\frac{\delta E_{x c}}{\delta \phi_{k}^{\dagger}\left(\mathbf{r}^{\prime}\right)}\right)+c . c .\right] \\
& \quad+\sum_{k} n_{k}(\mathbf{r}) \frac{\partial E_{x c}}{\partial \epsilon_{k}}=0 .
\end{aligned}
$$

Now one can use eq. (21):

$$
\begin{aligned}
& \left\{-\frac{\nabla^{2}}{2 m}+v_{s}(\mathbf{r})-\epsilon_{k}\right\} \int d^{3} r^{\prime} G_{k}\left(\mathbf{r}, \mathbf{r}^{\prime}\right) \\
& \quad \times\left[\phi_{k}\left(\mathbf{r}^{\prime}\right) v_{x c}\left(\mathbf{r}^{\prime}\right)-\frac{\delta E_{x c}}{\delta \phi_{k}^{\dagger}\left(\mathbf{r}^{\prime}\right)}\right] \\
& \quad=\phi_{k}(\mathbf{r}) v_{x c}(\mathbf{r})-\frac{\delta E_{x c}}{\delta \phi_{k}^{\dagger}(\mathbf{r})}-\left[\bar{v}_{k}-\bar{e}_{k}\right] \phi_{k}(\mathbf{r})
\end{aligned}
$$


and the conjugated equation to rewrite eq. (67) $\mathrm{as}^{70}$ :

$$
\begin{aligned}
0= & v_{s}(\mathbf{r})\left\{\left[\sum_{k} \phi_{k}^{\dagger}(\mathbf{r}) \int d^{3} r^{\prime} G_{k}\left(\mathbf{r}, \mathbf{r}^{\prime}\right)\right.\right. \\
& \left.\times\left(\phi_{k}\left(\mathbf{r}^{\prime}\right) v_{x c}\left(\mathbf{r}^{\prime}\right)-\frac{\delta E_{x c}}{\delta \phi_{k}^{\dagger}\left(\mathbf{r}^{\prime}\right)}\right)+c . c .\right] \\
& \left.+\sum_{k} n_{k}(\mathbf{r}) \frac{\partial E_{x c}}{\partial \epsilon_{k}}\right\} \\
= & \sum_{k} \phi_{k}^{\dagger}(\mathbf{r})\left\{\int d^{3} r^{\prime}\left[\frac{\nabla^{2}}{2 m}+\epsilon_{k}\right] G_{k}\left(\mathbf{r}, \mathbf{r}^{\prime}\right)\right. \\
& \times\left[\phi_{k}\left(\mathbf{r}^{\prime}\right) v_{x c}\left(\mathbf{r}^{\prime}\right)-\frac{\delta E_{x c}}{\delta \phi_{k}^{\dagger}\left(\mathbf{r}^{\prime}\right)}\right] \\
& \left.+\phi_{k}(\mathbf{r}) v_{x c}(\mathbf{r})-\frac{\delta E_{x c}}{\delta \phi_{k}^{\dagger}(\mathbf{r})}-\left[\bar{v}_{k}-\bar{e}_{k}\right] \phi_{k}(r)\right\} \\
& +c . c .+v_{s}(\mathbf{r}) \sum_{k} n_{k}(\mathbf{r}) \frac{\partial E_{x c}}{\partial \epsilon_{k}}
\end{aligned}
$$

Reordering eq. (69) leads to an exact reformulation of eq. (24):

$$
\begin{aligned}
v_{x c}(\mathbf{r})= & \frac{1}{2 n(\mathbf{r})}\left\{\sum_{k}\left[\phi_{k}^{\dagger}(\mathbf{r}) \frac{\delta E_{x c}}{\delta \phi_{k}^{\dagger}(\mathbf{r})}+n_{k}(\mathbf{r})\left[\bar{v}_{k}-\bar{e}_{k}\right]\right]\right. \\
& + \text { c.c. }-\sum_{k} \phi_{k}^{\dagger}(\mathbf{r}) \int d^{3} r^{\prime}\left[\frac{\nabla^{2}}{2 m}+\epsilon_{k}\right] G_{k}\left(\mathbf{r}, \mathbf{r}^{\prime}\right) \\
& \times\left[\phi_{k}\left(\mathbf{r}^{\prime}\right) v_{x c}\left(\mathbf{r}^{\prime}\right)-\frac{\delta E_{x c}}{\delta \phi_{k}^{\dagger}\left(\mathbf{r}^{\prime}\right)}\right]+\text { c.c. } \\
& \left.-v_{s}(\mathbf{r}) \sum_{k} n_{k}(\mathbf{r}) \frac{\partial E_{x c}}{\partial \epsilon_{k}}\right\}
\end{aligned}
$$

At first glance, this appears to be an even more complicated version of the OPM integral equation than eq. (24). In fact, the right-hand side of eq. (70) depends on $v_{x c}$, not only via $\bar{v}_{k}$ but also explicitly. However, the first term on the right-hand side of eq. (70) reduces exactly to the Slater potential in the exchange-only limit:

$$
v_{x}^{S}(\mathbf{r})=\frac{1}{2 n(\mathbf{r})} \sum_{k}\left[\phi_{k}^{\dagger}(\mathbf{r}) \frac{\delta E_{x}}{\delta \phi_{k}^{\dagger}(\mathbf{r})}+\text { c.c. }\right]
$$

so that this term dominates over the remaining contributions. This then immediately suggests that one neglects the second line on the right-hand side, leading to a second form (b) of the KLI approximation:

$$
\begin{aligned}
v_{x c}^{K L I b}(\mathbf{r})= & \frac{1}{2 n(\mathbf{r})} \\
& \times\left\{\sum_{k}\left[\phi_{k}^{\dagger}(\mathbf{r}) \frac{\delta E_{x c}}{\delta \phi_{k}^{\dagger}(\mathbf{r})}-n_{k}(\mathbf{r}) \bar{e}_{k}+c . c .\right]\right. \\
& \left.+2 \sum_{\epsilon_{k} \leq \epsilon_{F}} n_{k}(\mathbf{r}) \bar{v}_{k}-v_{s}(\mathbf{r}) \sum_{k} n_{k}(\mathbf{r}) \frac{\partial E_{x c}}{\partial \boldsymbol{\epsilon}_{k}}\right\}
\end{aligned}
$$

Yet another form of the OPM integral equation is obtained from eq. (70) by adding zero in the form:

$$
\begin{aligned}
0= & \frac{1}{2 n(\mathbf{r})}\left[\frac{\nabla^{2}}{2 m}+v_{s}(\mathbf{r})\right]\left\{\sum_{k} \phi_{k}^{\dagger}(\mathbf{r}) \int d^{3} r^{\prime} G_{k}\left(\mathbf{r}, \mathbf{r}^{\prime}\right)\right. \\
& \times\left[\phi_{k}\left(\mathbf{r}^{\prime}\right) v_{x c}\left(\mathbf{r}^{\prime}\right)-\frac{\delta E_{x c}}{\delta \phi_{k}^{\dagger}\left(\mathbf{r}^{\prime}\right)}\right]+c . c . \\
& \left.+\sum_{k} n_{k}(\mathbf{r}) \frac{\partial E_{x c}}{\partial \boldsymbol{\epsilon}_{k}}\right\}
\end{aligned}
$$

and subsequently using the KS equations [eq. (9)]:

$$
\begin{aligned}
v_{x c}(\mathbf{r})= & \frac{1}{2 n(\mathbf{r})}\left\{\sum_{k}\left[\phi_{k}^{\dagger}(\mathbf{r}) \frac{\delta E_{x c}}{\delta \phi_{k}^{\dagger}(\mathbf{r})}+n_{k}(\mathbf{r})\left[\bar{v}_{k}-\bar{e}_{k}\right]\right]\right. \\
& + \text { c.c. }+\sum_{k} \int d^{3} r^{\prime} \frac{\nabla}{m}\left[\left(\nabla \phi_{k}^{\dagger}(\mathbf{r})\right) G_{k}\left(\mathbf{r}, \mathbf{r}^{\prime}\right)\right] \\
& \times\left[\phi_{k}\left(\mathbf{r}^{\prime}\right) v_{x c}\left(\mathbf{r}^{\prime}\right)-\frac{\delta E_{x c}}{\delta \phi_{k}^{\dagger}\left(\mathbf{r}^{\prime}\right)}\right]+\text { c.c. } \\
& \left.+\frac{\nabla^{2}}{2 m} \sum_{k} n_{k}(\mathbf{r}) \frac{\partial E_{x c}}{\partial \epsilon_{k}}\right\}
\end{aligned}
$$

Eq. (74) is the direct extension of the form of the OPM integral equation given in ref. 62. Neglecting the second line in eq. (74) leads to a third form (c) of the KLI approximation:

$$
\begin{aligned}
v_{x c}^{K L I c}(\mathbf{r})= & \frac{1}{2 n(\mathbf{r})} \\
& \times\left\{\sum_{k}\left[\phi_{k}^{\dagger}(\mathbf{r}) \frac{\delta E_{x c}}{\delta \phi_{k}^{\dagger}(\mathbf{r})}+n_{k}(\mathbf{r})\left[\bar{v}_{k}-\bar{e}_{k}\right]\right]\right. \\
& \left.+ \text { c.c. }+\frac{\nabla^{2}}{2 m} \sum_{k} n_{k}(\mathbf{r}) \frac{\partial E_{x c}}{\partial \epsilon_{k}}\right\}
\end{aligned}
$$

Note that the quality of the form of eq. (75) is underlined by the fact that the spatial average of 
the second line in eq. (74) vanishes ${ }^{62}$ :

$$
\begin{aligned}
\int d^{3} r & \sum_{k} \int d^{3} r^{\prime} \frac{\nabla}{m}\left[\left(\nabla \phi_{k}^{\dagger}(\mathbf{r})\right) G_{k}\left(\mathbf{r}, \mathbf{r}^{\prime}\right)\right] \\
\times & {\left[\phi_{k}\left(\mathbf{r}^{\prime}\right) v_{x c}\left(\mathbf{r}^{\prime}\right)-\frac{\delta E_{x c}}{\delta \phi_{k}^{\dagger}\left(\mathbf{r}^{\prime}\right)}\right]+\text { c.c. }=0 }
\end{aligned}
$$

Both eqs. (72) and (75) provide explicit density functionals for the energy scale $\Delta \bar{\epsilon}$. It is not clear whether there exist further forms of KLI-type approximations [which differ nontrivially from eqs. (62), (72), and (75)] and which of these forms is most appropriate. Moreover, the basic physical concept behind the closure approximation and the very fact that so many different versions of the KLI concept can be found for eigenvalue-dependent $E_{x c}$ suggest ignoring the $\partial E_{x c} / \partial \epsilon_{k}$ contribution completely.

This argument is supported by an analysis of the asymptotic behavior of the KLI potential for finite systems. Within the x-only limit, that is, for the functional (8), all three KLI forms coincide and reproduce the exact asymptotic form of $v_{x}$, eq. (28). ${ }^{50}$ On the other hand, if $E_{x c}$ also depends on the eigenvalues of unoccupied orbitals [as the functional eq. (46)], the crucial contribution:

$$
\sum_{k} \frac{n_{k}(\mathbf{r})}{n(\mathbf{r})} \frac{\partial E_{x c}}{\partial \boldsymbol{\epsilon}_{k}}
$$

is expected to diverge for large $\mathbf{r}$, at least in the general case. Consequently, the three versions of the KLI potential discussed in this section will also diverge for large $\mathbf{r}$, in contrast to the exact OPM potential. For the relativistic exchange, which depends only on the eigenvalues of the occupied levels, this term approaches a constant, so that neither of the aforementioned forms of the KLI approximation leads to the correct asymptotic behavior of the exchange potential of closed-subshell atoms. ${ }^{71}$ For this latter potential, a complete neglect of the $\partial E_{x c} / \partial \epsilon_{k}$ contribution yielded very accurate results. It remains to be verified whether an analogous statement applies to correlation functionals like eq. (46).

\section{Illustrative Results}

\section{EXCHANGE-ONLY RESULTS}

OPM results for atoms obtained within the $\mathrm{x}$ only limit have been discussed extensively in the literature. ${ }^{25,45,50,61-64,68,71}$ We thus restrict ourselves to providing an elementary comparison between orbital-dependent exchange and conventional density functionals (and to strictly nonrelativistic results). In Tables I and II, the total ground-state energies of closed-subshell atoms are listed, comparing exact OPM, KLI [which is unique

\begin{tabular}{|c|c|c|c|c|c|}
\hline & \multirow{2}{*}{$\begin{array}{l}E_{\text {tot }} \\
\text { OPM }\end{array}$} & \multicolumn{4}{|c|}{$E_{t o t}-E_{t o t}^{\mathrm{OPM}}$} \\
\hline & & KLI & LDA & GGA & $\mathrm{HF}$ \\
\hline $\mathrm{He}$ & -2861.7 & 0.0 & 138.0 & 6.5 & 0.0 \\
\hline $\mathrm{Be}$ & -14572.4 & 0.1 & 349.1 & 18.2 & -0.6 \\
\hline $\mathrm{Ne}$ & -128545.4 & 0.6 & 1054.7 & -23.5 & -1.7 \\
\hline $\mathrm{Mg}$ & -199611.6 & 0.9 & 1362.8 & -0.5 & -3.1 \\
\hline Ar & -526812.2 & 1.7 & 2294.8 & 41.2 & -5.3 \\
\hline $\mathrm{Ca}$ & -676751.9 & 2.2 & 2591.8 & 25.7 & -6.3 \\
\hline $\mathrm{Zn}$ & -1777834.4 & 3.7 & 3924.5 & -252.6 & -13.8 \\
\hline $\mathrm{Kr}$ & -2752042.9 & 3.2 & 5176.8 & -18.4 & -12.0 \\
\hline $\mathrm{Sr}$ & -3131533.4 & 3.6 & 5535.4 & -8.8 & -12.2 \\
\hline $\mathrm{Pd}$ & -4937906.0 & 4.5 & 6896.0 & -65.2 & -15.0 \\
\hline $\mathrm{Cd}$ & -5465114.4 & 6.0 & 7292.6 & -31.9 & -18.7 \\
\hline $\mathrm{Xe}$ & -7232121.1 & 6.1 & 8463.8 & 54.9 & -17.3 \\
\hline $\mathrm{Ba}$ & -7883526.6 & 6.5 & 8792.5 & 15.7 & -17.3 \\
\hline $\mathrm{Yb}$ & -13391416.3 & 10.0 & 10505.6 & -852.4 & -39.9 \\
\hline $\mathrm{Hg}$ & -18408960.5 & 9.1 & 13040.4 & -221.5 & -31.0 \\
\hline $\mathrm{Rn}$ & -21866745.7 & 8.5 & 14424.3 & 8.3 & -26.5 \\
\hline
\end{tabular}

TABLE I.

Self-consistent x-Only Ground-State Energies of Unpolarized Closed-Subshell Atoms. ${ }^{\text {a }}$

${ }^{\text {a }}$ OPM energies in comparison with $\mathrm{KLI}$, LDA, and PW91-GGA ${ }^{33}$ as well as HF results (all energies in millihartrees). 


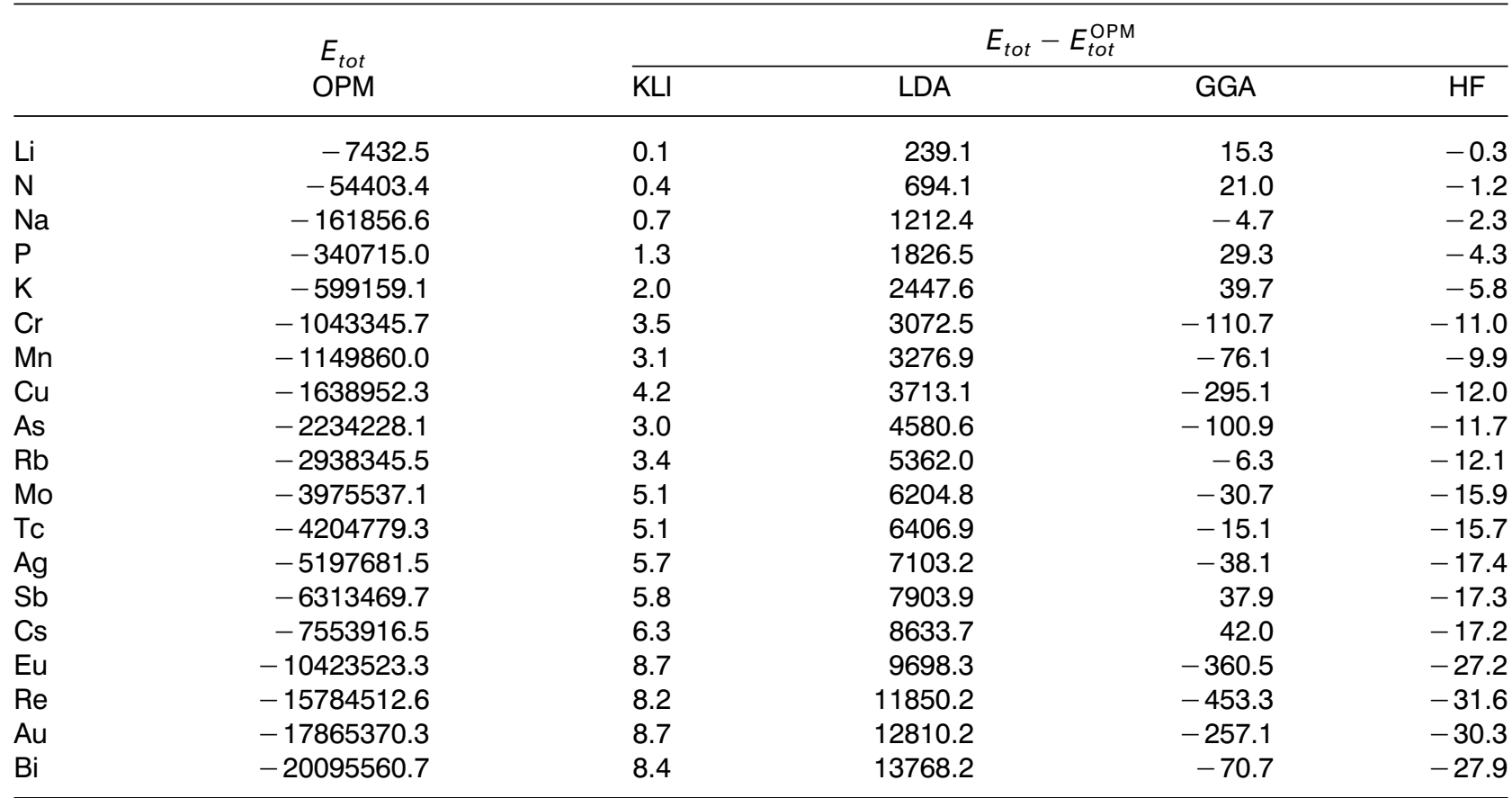

${ }^{a}$ OPM energies in comparison with KLI, LDA, and PW91-GGA ${ }^{33}$ as well as spin-unrestricted HF results (all energies in millihartrees).

for the exchange in eq. (8)], LDA, and PW91-GGA ${ }^{33}$ results]. The OPM numbers have been produced on radial meshes of 1600 mesh points, so that the error in the $\mathrm{x}$-only virial relation, ${ }^{80}$ which is a measure of the numerical accuracy, has been reduced to 0.14 mhartree $(\mathrm{mH})$ even for the heaviest atom, radon (for details compare with ref 64 ). The OPM numbers may thus serve as reference values (an error on the 0.1-mH level in the code used for ref. 64 has been corrected).

Comparing the OPM with its KLI approximation it is obvious that the KLI scheme produces highly accurate results throughout the periodic table. The differences between OPM and KLI ground-state energies are below the $10-\mathrm{mH}$ level, suggesting the KLI approximation to be an adequate substitute for the much more involved full OPM in the case of the exchange functional. The KLI energies are always above the OPM results, consistent with the fact that the OPM yields that $v_{x}$ value which minimizes the energy functional at hand. On the other hand, the x-only OPM groundstate energies are always slightly above the corresponding HF values, which demonstrates the reduced variational freedom in the former approach: Whereas both procedures minimize the same energy expression, the OPM restricts $v_{x}$ to be a multiplicative potential. The nonlocal HF exchange potential thus produces a somewhat lower minimum. ${ }^{81}$ From a physical point of view, however, these differences are irrelevant, thus supporting the concept of multiplicative effective potentials. This conclusion is supported by Table III, in which we compare the first ionization potentials (IPs) of atoms with spherical density. For all atoms considered, the OPM, KLI, and HF values are essentially identical.

The LDA total energies, on the other hand, deviate substantially from the exact OPM numbers. At first glance, these errors seem completely unacceptable for a method aiming at the description of chemical binding. One has to note, however, that these large errors originate mainly from the chemically inactive core electrons. In fact, given its errors for total energies, the LDA is remarkably accurate for the physically more relevant energy differences. This is illustrated in Table III, which shows that the LDA underestimates the exact $x$ only IPs for light atoms and overestimates them for heavy atoms. Nevertheless, although in the IPs the errors of the LDA are reduced to the order of 5 to $50 \mathrm{mH}$, this is still not sufficient to achieve chemical accuracy.

Finally, focusing on the PW91-GGA, one notices a clear improvement over the LDA for ground-state energies. However, as the sign-indefiniteness of 
TABLE III. First Ionization Potentials within x-Only Limit. ${ }^{a}$

\begin{tabular}{|c|c|c|c|c|c|}
\hline Atom & OPM & KLI & LDA & GGA & $\mathrm{HF}$ \\
\hline $\mathrm{He}$ & 862 & 862 & 811 & 866 & 862 \\
\hline $\mathrm{Be}$ & 295 & 295 & 281 & 301 & 296 \\
\hline $\mathrm{Mg}$ & 242 & 242 & 238 & 254 & 243 \\
\hline $\mathrm{Ca}$ & 188 & 188 & 189 & 200 & 188 \\
\hline $\mathrm{Sr}$ & 171 & 171 & 174 & 184 & 171 \\
\hline $\mathrm{Ba}$ & 152 & 151 & 155 & 164 & \\
\hline $\mathrm{Cu}$ & 231 & 229 & 278 & 285 & 236 \\
\hline $\mathrm{Ag}$ & 215 & 214 & 251 & 256 & 218 \\
\hline$A u$ & 216 & 214 & 254 & 258 & 218 \\
\hline $\mathrm{Li}$ & 196 & 196 & 185 & 200 & 196 \\
\hline $\mathrm{Na}$ & 181 & 181 & 179 & 191 & 182 \\
\hline $\mathrm{K}$ & 147 & 147 & 149 & 157 & 147 \\
\hline $\mathrm{Rb}$ & 137 & 137 & 141 & 149 & 138 \\
\hline Cs & 123 & 123 & 128 & 134 & 123 \\
\hline $\mathrm{Zn}$ & 276 & 276 & 310 & 320 & 281 \\
\hline $\mathrm{Cd}$ & 252 & 251 & 282 & 289 & \\
\hline $\mathrm{Hg}$ & 248 & 248 & 281 & 287 & \\
\hline
\end{tabular}

${ }^{\mathrm{a}}$ OPM energies in comparison with KLI, LDA, PW91-GGA, ${ }^{33}$ and HF results (all energies in millihartrees).

the error of the GGA already indicates, this quality, to some extent, relies on a subtle error cancelation. As a consequence, the same degree of improvement is not found for IPs: the PW91-GGA consistently increases the IP, so that it improves over the LDA for light atoms, but produces larger errors than the LDA for the heavy systems. Neither alternative forms of the GGA (such as the Becke GGA ${ }^{28}$ ) nor other conventional density functionals (such as, e.g., the weighted density approximation ${ }^{82,83}$ ) lead to consistently superior results, so that the use of the exact exchange presently seems imperative for many purposes.

The exchange potentials of neon and zinc are plotted in Figures 1 and 2. While the LDA and GGA exchange potentials are rather smooth functions of $r$, the exact exchange produces marked "bumps," reflecting the shell structure of the atoms. As a consequence, the OPM shells are somewhat more localized than the LDA/GGA results, as can be seen in Figures 3 and 4 for the $l$ shell of neon and the $m$ shell of zinc. The KLI approximation also generates shell effects in $v_{x}$, but not as pronounced as the full OPM. The radial density obtained with the KLI approximation, on the other hand, is very close to the OPM result. Figures 1 and 2 also demonstrate the asymptotic behavior of $v_{x}$. As stated in eq. (28), the exact $v_{x}$ falls off like $-e^{2} / r$, and the KLI approximation preserves this property. The LDA and GGA potentials, on the other hand, decay exponentially due to the fact that the self-interaction of the outermost electron is not appropriately eliminated by these functionals.

The exact x-only OPM has also been formulated for plane-wave pseudopotential calculations. ${ }^{75}$ This scheme has been applied to $\mathrm{Si}, \mathrm{C}, \mathrm{GaN}$, and $\mathrm{InN},{ }^{67}$ using the results for a detailed comparison of the

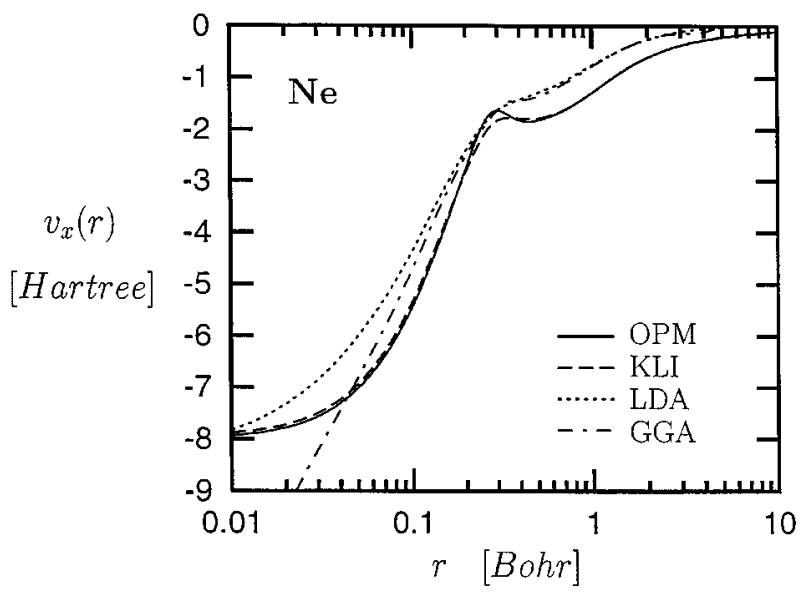

FIGURE 1. X-Only exchange potential of neutral $\mathrm{Ne}$. Self-consistent OPM, KLI, LDA, and PW91-GGA results. 


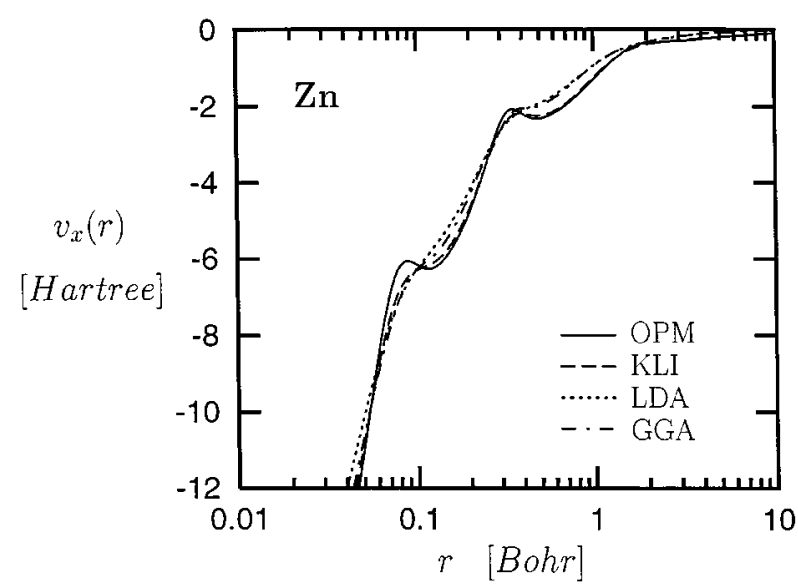

FIGURE 2. Data same as Figure 1, for neutral Zn.

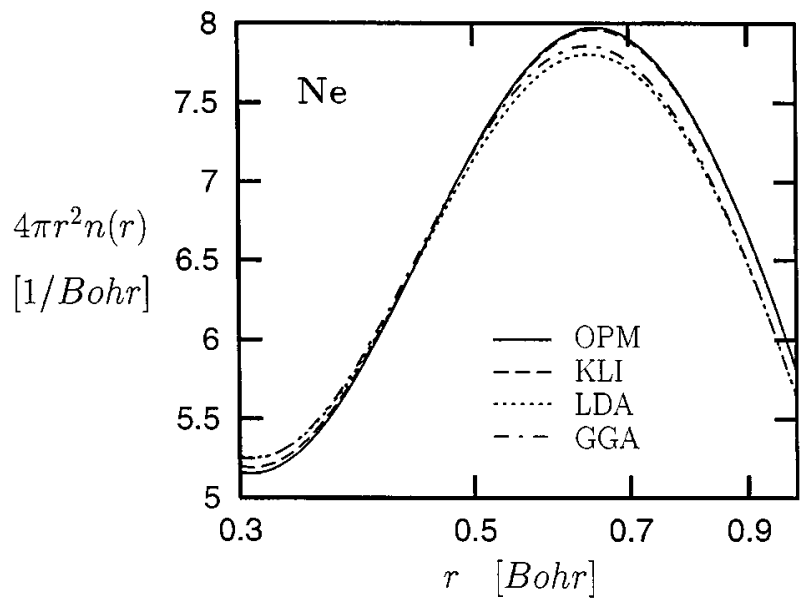

FIGURE 3. X-Only ground-state density of neutral $\mathrm{Ne}$. Self-consistent OPM, KLI, LDA, and PW91-GGA results.

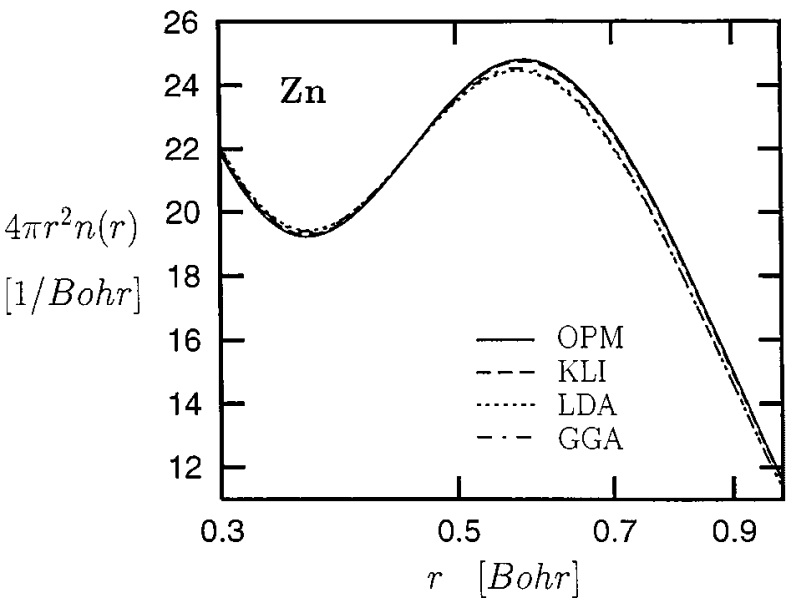

FIGURE 4. Data same as Figure 3 , for neutral Zn. exact exchange with the LDA and various GGAs. As in the case of atoms, one finds that the LDA underestimates the spatial variations in $v_{x}$, whereas the GGA improves $v_{x}$ in the bonding region, at the price of introducing artificial structures close to the nuclei. Again, similar to the situation for atoms, ${ }^{64}$ the LDA exchange energies deviate by roughly $6 \%$ from the exact values. ${ }^{67}$ The GGAs considered in ref. 67 , on the other hand, consistently produce errors of the order of $1 \%$, demonstrating why GGAs improve the cohesive properties of many solids over the LDA. Furthermore, both the total and the exchange energy of the $\mathrm{x}$-only OPM calculation for $\mathrm{Si}$ are in close agreement with the corresponding HF values, completely in line with the observations for atoms.

\section{EXACT EXCHANGE WITH LDA/GGA FOR CORRELATION}

An all-electron OPM scheme for solids within the atomic-sphere approximation (ASA) has been put forward in ref. 65, combining the exact exchange with the LDA for correlation (using both the LMTO and the KKR framework). Applications to $\mathrm{MgO}, \mathrm{CaO}, \mathrm{MnO}, \mathrm{Si}, \mathrm{Ge}$, and $\mathrm{C}^{65}$ show that the exact exchange leads to more localized valence electrons than the LDA for solids also. The resulting band gaps are larger than the corresponding LDA values and thus agree better with experiment. This basic observation is confirmed by plane-wave pseudopotential calculations combining exact exchange and LDA correlation ${ }^{67}$ (but not utilizing the ASA), in which the OPM band gaps of Si, C, GaN, and InN have been found to be roughly $1 \mathrm{eV}$ larger than the LDA gaps. As in the case of atomic IPs and electron affinities (EAs), the proper cancelation of electron self-interaction turns out to be important for obtaining realistic band structures. Although one would expect an equivalent orbital-dependent treatment of $E_{c}$ to reduce the gaps, ${ }^{65}$ this nevertheless seems to indicate progress.

The OPM has also been applied to solids within the KLI approximation, ${ }^{66}$ again using the exact $E_{x}$ with the LDA for correlation and pseudopotentials to represent the core electrons. Consistent with the earlier calculations utilizing the full OPM, this method predicts increased band gaps. The higher efficiency of the KLI approximation, however, also permits determination of the structure and energetics of solids. It is found that lattice constants are increased compared with LDA results, which improves the agreement with experiment for $\mathrm{Si}$ and 
Ge, but slightly worsens the agreement for GaAs. The cohesive energies, on the other hand, come out much too small, so the investigators concluded $^{66}$ that the LDA is not well suited for use with the exact exchange. The last problem can be resolved partially by using a GGA for correlation, ${ }^{66}$ but at the price of a reducing the quality of indirect gaps. In conclusion, it seems that these applications all indicate the need for an adequate orbital-dependent correlation functional to match the exact $E_{x}$.

\section{ORBITAL-DEPENDENT CORRELATION ENERGY}

The most simple orbital-dependent correlation functional available for use with the exact exchange is the CS expression. ${ }^{55}$ The CS functional produces rather accurate correlation energies for light atoms. ${ }^{56}$ However, the corresponding IPs are not actually superior to those obtained with GGAs (for exchange and correlation) and, whereas the CS functional predicts most negative ions to be bound, the calculated EAs are not very reliable. ${ }^{56}$ This is underlined by Table IV in which a number of atomic EAs obtained by combining the exact exchange with various correlation functionals are given (for open-shell systems the calculations have been restricted to the spherical average; the correlation energies have been evaluated perturbatively, using the self-consistent $\mathrm{x}$-only orbitals). From Table IV it is obvious that the CS functional does not generate EAs that are more accurate than those found with conventional density function-

TABLE IV.

Electron Affinities of First- and Second-Row Atoms. ${ }^{a}$

\begin{tabular}{lrrrr}
\hline & LDA & GGA & CS & Exp. \\
\hline $\mathrm{Li}$ & 27 & 16 & 15 & 23 \\
$\mathrm{~B}$ & 14 & 6 & -2 & 10 \\
$\mathrm{C}$ & 48 & 39 & 28 & 46 \\
$\mathrm{O}$ & 33 & 15 & 17 & 54 \\
$\mathrm{~F}$ & 101 & 84 & 82 & 125 \\
$\mathrm{Na}$ & 25 & 15 & 15 & 20 \\
$\mathrm{Al}$ & 20 & 16 & 6 & 16 \\
$\mathrm{Si}$ & 58 & 53 & 40 & 51 \\
$\mathrm{P}$ & 29 & 13 & 22 & 27 \\
$\mathrm{~S}$ & 80 & 68 & 65 & 76 \\
$\mathrm{Cl}$ & 140 & 128 & 122 & 133 \\
\hline \multicolumn{5}{c}{ a Exact exchange in combination with LDA, PW91-GGA, and } \\
\multicolumn{5}{c}{ CS correlation (evaluated perturbatively with self-consistent } \\
\multicolumn{5}{c}{ energies in millihartrees). }
\end{tabular}

als. In fact, for the EAs shown, the LDA correlation seems to produce the most accurate results (throughout this work the form of ref. 9 is used for the LDA correlation energy). This failure of the CS functional is reflected by the fact that the CS correlation potential has little in common with the exact $v_{c}$ in the few cases in which the latter is known. ${ }^{84}$ This is shown in Figure 5 where we compare the exact $v_{c}$ of neutral neon ${ }^{84}$ with the potentials obtained by insertion of self-consistent x-only orbitals into the CS functional, the LDA, and the PW91GGA. Even the sign of the CS potential is in error in most regions (in particular, in the most relevant valence region). Figure 5 clearly demonstrates the limitations of both semiempirical and electrongas-based representations of $v_{c}$.

Although one would hope to overcome this problem with the functional eq. (46) its self-consistent application is complicated by the fact that the rigorous solution of the corresponding OPM equation [eq. (47)] requires knowledge of the quadratic response function [eq. (51)]. To provide some initial idea of the performance of this functional we evaluated eq. (46) perturbatively, using $\mathrm{x}$-only OPM orbitals as input. Because the correlation potential gives only a minor contribution to the total $v_{s}$, the resulting correlation energies should be rather close to the corresponding selfconsistent values.

In Table $\mathrm{V}$, we list the $E_{c}^{(2)}$ obtained for the helium isoelectronic series in comparison to conventional DFT, MP2, ${ }^{85}$ and variational (i.e., exact) ${ }^{86}$ results. As is immediately obvious from the table, $E_{c}^{(2)}$ is consistently closer to the true correlation energies than any of the conventional density func-

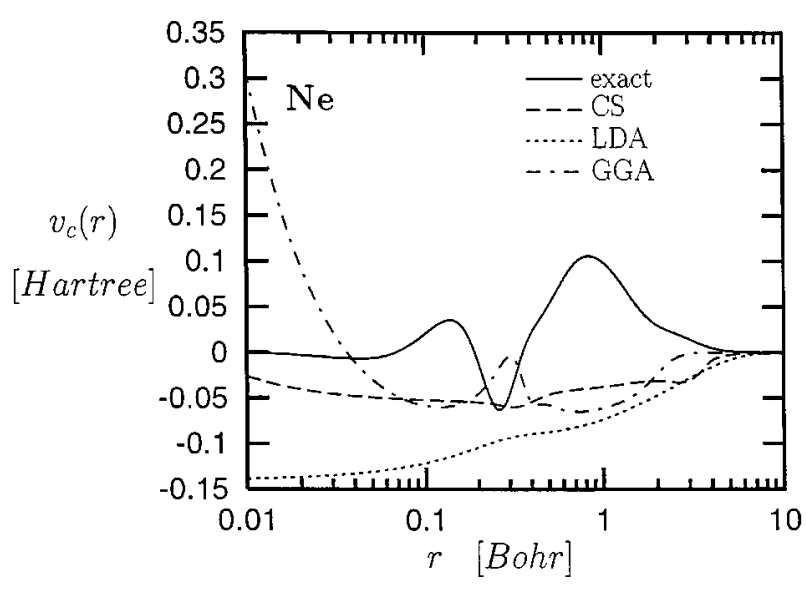

FIGURE 5. Correlation potential of neutral Ne. CS, LDA, and PW91-GGA potentials in comparison with exact result by C. J. Umrigar and collaborators. ${ }^{84}$ 
TABLE V.

Correlation Energies $\left(-E_{c}\right)$ of He Isoelectronic Series. ${ }^{a}$

\begin{tabular}{lllllr}
\hline Ion & LDA & GGA & CS & OPM & MP2 \\
\hline $\mathrm{He}$ & 112.8 & 45.9 & 41.6 & 48.21 & 37.14 \\
$\mathrm{Ne}^{8+}$ & 203.0 & 61.7 & 40.6 & 46.81 & 42.04 \\
$\mathrm{Ca}^{18+}$ & 243.3 & 67.7 & 35.9 & 46.69 & 45.69 \\
$\mathrm{Zn}^{28+}$ & 267.2 & 71.3 & 33.2 & 46.67 & 45.37 \\
$\mathrm{Zr}^{38+}$ & 284.4 & 74.0 & 31.4 & 46.66 & 45.71 \\
$\mathrm{Sn}^{48+}$ & 297.7 & 76.0 & 30.0 & 46.65 & 45.88 \\
$\mathrm{Nd}^{58+}$ & 308.7 & 77.8 & 29.0 & 46.34 \\
$\mathrm{Yb}^{68+}$ & 318.0 & 79.3 & 28.2 & 46.64 & 46.42 \\
$\mathrm{Hg}^{78+}$ & 326.1 & 80.6 & 27.6 & 46.63 & 46.47 \\
$\mathrm{Th}^{88+}$ & 333.2 & 81.7 & 27.0 & 46.62 & 46.51 \\
$\mathrm{Fm}^{98+}$ & 339.6 & 82.8 & 26.0 & 46.62 & 46.53 \\
\hline
\end{tabular}

${ }^{\mathrm{a}} \mathrm{LDA},{ }^{9} \mathrm{PW} 91-\mathrm{GGA},{ }^{33} \mathrm{CS},{ }^{55}$ conventional MP2, ${ }^{85}$ and exact variational energies ${ }^{86}$ in comparison with correlated OPM results, obtained by perturbative evaluation of the functional [eq. (46)] using x-only OPM orbitals (all energies in millihartrees).

tionals. While it is well known that the LDA completely misrepresents atomic correlation energies, PW91-GGA also overestimates the true $E_{c}$ for high $Z$ by almost a factor of 2 . The CS functional, on the other hand, is very close to the correct result for helium (to which its parameters have been adjusted), but dramatically underestimates $E_{c}$ for high $Z$. The only DFT expression that shows the correct scaling with $Z$ (i.e., approaches a constant of the correct size) is the function, eq. (46). In fact, although $E_{c}^{(2)}$ is only moderately accurate for neutral helium, its error reduces to about $1 \mathrm{mH}$ already for $\mathrm{Ne}^{8+}$. Finally, in the limit of large $\mathrm{Z}$, it only deviates by $0.05 \mathrm{mH}$ from the $1 / Z$ expansion formula of Davidson and coworkers, ${ }^{86}$ which is based on variational calculations.

$E_{c}^{(2)}$ is roughly $25 \%$ larger than the conventional MP2 result for neutral helium, but approaches the MP2 data rapidly with increasing $Z$. This behavior mainly originates from the fact that OPM calculations lead to a Rydberg series of excited levels, even for neutral atoms. ${ }^{81}$ This can be verified by separating those correlation contributions that involve virtual excitations into the Rydberg levels from the correlation energy due to excitations into the positive continuum states. For helium, the latter amounts to $-39.8 \mathrm{mH}$, which compares rather well with the MP2 value of $-37.1 \mathrm{mH}$. With increasing $Z$, on the other hand, the sum of the nuclear and the Hartree potential more and more dominates over the exchange potential. Consequently the Rydberg states resulting from the HF and the OPM potentials become more and more similar, which explains why the corresponding $E_{c}$ approach each other for high $\mathrm{Z}$. Compared with the exact $E_{c}$ of helium, $E_{c}^{(2)}$ is $14 \%$ too large, whereas the MP2 value is $12 \%$ too small. It is not clear at the moment to what extent these discrepancies are transferred into energy differences like IPs and EAs.

Results for the most simple negative ion, the $\mathrm{H}^{-}$, are given in Table VI. In this special case the correlation energy directly enters the calculation of the EA, without involving a correlation energy difference. Several combinations of the exact exchange with approximate correlation functionals are analyzed. As for the helium atom one finds that the functional [eq. (46)] overestimates the true correlation energy obtained variationally ${ }^{87}$ : Its error increases to almost $15 \mathrm{mH}$. Consequently, its absolute deviation from the exact EA is larger than that found with the PW91-GGA for $E_{c}(5 \mathrm{mH})$ and

TABLE VI. Correlation Energy and Electron Affinity of $\mathrm{H}^{-}$Ion. ${ }^{\mathrm{a}}$

\begin{tabular}{lcc}
\hline \multicolumn{1}{c}{ Method } & $-E_{c}$ & EA \\
\hline Exact (ref. 87) & 39.8 & 27.8 \\
OPM $\left(E_{x}+E_{c}^{\mathrm{LDA}}\right)$ & 75.7 & 62.6 \\
OPM $\left(E_{x}+E_{c}^{\mathrm{GGA}}\right)$ & 35.5 & 22.8 \\
OPM $\left(E_{x}+E_{c}^{\mathrm{CS}}\right)$ & 31.2 & 18.9 \\
OPM $\left(E_{x}+E_{c}^{(2)}\right)$ & 54.6 & 42.6 \\
MP2 (ref. 91) & & \\
QCI (ref. 91) & 27.3 & 15.2 \\
\hline
\end{tabular}

${ }^{a}$ Exact exchange in combination with LDA, ${ }^{9}$ PW91-GGA, ${ }^{33}$ $\mathrm{CS}^{55}$ and eq. (46) (evaluated perturbatively using x-only OPM orbitals) for correlation in comparison with MP2, QCI, and exact variational energies (all energies in millihartrees). 
TABLE VII.

\section{Spectroscopic Parameters of $\mathrm{H}_{2}{ }^{\text {a }}$}

\begin{tabular}{lccc}
\hline & $\begin{array}{c}r_{e} \\
(\text { bohr })\end{array}$ & $\begin{array}{c}-E_{b} \\
(\mathrm{eV})\end{array}$ & $\begin{array}{c}\omega_{e} \\
\left(\mathrm{~cm}^{-1}\right)\end{array}$ \\
\hline Exp. & 1.40 & 4.75 & 4400 \\
Exact (ref. 92) & 1.41 & 4.75 & 4483 \\
LDA & 1.45 & 4.91 & 4185 \\
GGA (ref. 93) & 1.42 & 4.56 & 4332 \\
OPM ( $\left.E_{x}+E_{c}^{\mathrm{LDA}}\right)$ & 1.37 & 5.02 & 4833 \\
OPM $\left(E_{x}+E_{c}^{C S}\right)$ & 1.38 & 4.66 & 4659 \\
OPM (Ex $\left.+E_{c}^{(2)}\right)$ & 1.42 & 4.99 & 4317 \\
HF & 1.38 & 3.64 & 4622 \\
MP2 (ref. 89) & 1.39 & 4.52 & \\
MP4 (ref. 89) & 1.40 & 4.70 & \\
QCISD (ref. 38) & 1.41 & 4.22 & 4367 \\
\hline
\end{tabular}

${ }^{\mathrm{a}} E_{b}$ includes the zero point energy, $E_{b}=-D_{e}-\hbar \omega_{e} / 2$. DFT and conventional $a b$ initio results in comparison with variational (exact) and experimental data.

the CS functional ( $9 \mathrm{mH})$. Only the LDA correlation completely misrepresents the EA. For $\mathrm{H}^{-}$, the difference between $E_{c}^{(2)}$ and the MP2 correlation energy increases to roughly a factor of two, although, in this case, Rydberg states are not present in the OPM. Again, $E_{c}^{(2)}$ and $E_{c}^{M P 2}$ bracket the exact $E_{c}$, their absolute errors being very similar.

A corresponding comparison for the hydrogen dimer is reported in Table VII and Figure 6. $E_{c}^{(2)}$

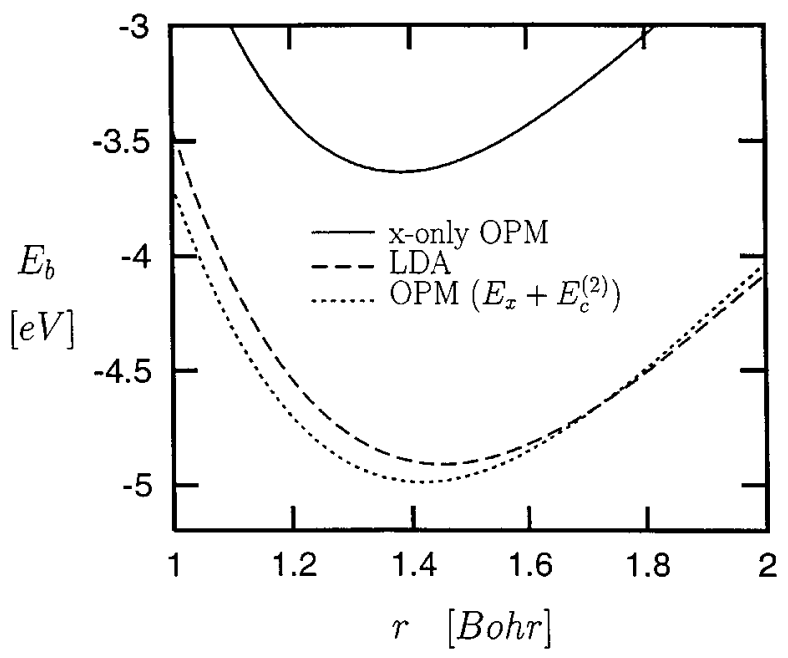

FIGURE 6. Energy surface of $\mathrm{H}_{2}$. Self-consistent LDA and $x$-only OPM calculations in comparison with correlated OPM data, obtained by perturbative evaluation of the function [eq. (46)] using x-only OPM orbitals. produces clearly more realistic data than the LDA for the equilibrium bond length and the zero point energy. In contrast to the PW91-GGA, which underestimates the binding energy, $E_{c}^{(2)}$ slightly increases $E_{b}$ compared with the LDA value, so that it does not improve over the LDA in this respect. The overall accuracy of $E_{c}^{(2)}$ is rather similar to that of the PW91-GGA. Although the effect of basis set truncation ${ }^{88}$ cannot be ignored completely in the comparison with the conventional, correlated $a b$ initio methods (in particular for the QCISD-values of ref. 38), $E_{c}^{(2)}$ nevertheless again seems to give a larger correlation energy than the conventional MP2 calculation ${ }^{89}$ (50 $\mathrm{mH}$ vs. $32 \mathrm{mH}$ at the equilibrium bond length). In fact, the difference of $18 \mathrm{mH}$ between the correlated OPM and the MP2 energy is not too far from the 11-mH deviation that one finds for neutral helium. As for helium, $E_{c}^{(2)}$ overestimates the correlation energy of $\mathrm{H}_{2}$ as much as MP2 underestimates it. The fourth-order Møller-Plesset (MP4) result could thus indicate that a corresponding fourth-order expansion in terms of the KS Hamiltonian is required to obtain very accurate spectroscopic constants for $\mathrm{H}_{2}$.

In Table VII we also give results of self-consistent calculations in which the exact exchange has been combined with the LDA and the CS functional for correlation. Both combinations do not actually improve results over the standard LDA calculation: In particular, the error of the standard LDA in the bond length (which is less sensitive to details of the xc-functional than the energy surface) is by far overcorrected by both orbital-dependent xc-functionals. Moreover, while the correlation LDA even increases the dissociation energy, the CS functional reduces it too much. These data demonstrate that the exact $E_{x}$ should not be used with the LDA for correlation, consistent with the findings in ref. 66, and the same statement seems to apply to the CS functional. Whether a GGA for correlation can serve as an adequate counterpart for the exact exchange in molecular calculations is not yet clear. In view of the limited quality of the GGA correlation potential, ${ }^{84}$ however, it seems that, ultimately, an orbital-dependent form for $E_{c}$ like eq. (46) is required, rather than a standard density functional.

\section{Acknowledgments}

The authors thank A. Facco Bonetti for valuable help (in particular, for providing the $E_{c}^{(2)}$ in Table 
V), and C. J. Umrigar for making his data of the exact neon correlation potential available to us.

\section{References}

1. Dreizler, R. M.; Gross, E. K. U. Density Functional Theory; Springer: Berlin, 1990.

2. Parr, R. G.; Yang, W. Density-Functional Theory of Atoms and Molecules; Oxford University Press: New York, 1989.

3. Jones, R. O.; Gunnarsson, O. Rev Mod Phys 1989, 61, 689.

4. Kohn W.; Sham, L. J. Phys Rev 1965, 140A, 1133.

5. von Barth, U.; Hedin, L. J Phys C 1972, 5, 1629.

6. Gunnarsson, O.; Lundqvist, B. I. Phys Rev B 1976, 13, 4274.

7. Ceperley, D. M.; Alder, B. J. Phys Rev Lett 1980, 45, 566.

8. Ortiz, G.; Ballone, P. Phys Rev B 1994, 50, 1391.

9. Vosko, S. H.; Wilk, L.; Nusair, M. Can J Phys 1980, 58, 1200.

10. Perdew, J. P.; Zunger, A. Phys Rev B 1981, 23, 5048.

11. Perdew, J. P.; Wang, Y. Phys Rev B 1992, 45, (13), 244.

12. Moruzzi, V. L.; Williams, A. R.; Janak, J. F. Phys Rev B 1977, 15, 2854; and Moruzzi, V. L.; Janak, J. F.; Williams, A. R. Calculated Electronic Properties of Metals; Pergamon: New York, 1978.

13. Martins, J. L.; Buttet, J.; Car, R. Phys Rev B 1985, 31, 1804.

14. Tong, B. Y. Phys Rev. A 1971, 4, 1375.

15. Jones R. O.; Gunnarsson, O. Phys Rev Lett 1985, 55, 107.

16. Shore, H. B.; Rose, J. H.; Zaremba, E. Phys Rev B 1977, 15, 2858.

17. Stoll, H.; Pavlidou, C. M. E.; Preuss, H. Theor Chim Acta 1978, 149, 143.

18. Vosko, S. H.; Wilk, L. J Phys B 1983, 16, 3687.

19. Ma, S.-K.; Brueckner, K. A. Phys Rev 1968, 165, 18.

20. Herman, F.; Van Dyke, J. P.; Ortenburger, I. B. Phys Rev Lett 1969, 22, 807; Herman, F.; Ortenburger, I. B.; Van Dyke, J. P. Int J Quant Chem 1970, IIIS, 827.

21. Sham, L. J. In P. M. Marcus, J. F. Janak, and A. R. Williams (Eds.), Computation Methods in Band Theory; Plenum: New York, 1971; p. 458.

22. Geldart, D. J. W.; Rasolt, M. Phys Rev B 1976, 13, 1477.

23. Kleinman, L.; Lee, S. Phys Rev B 1988, 37, 4634.

24. Engel, E.; Vosko, S. H. Phys Rev B 1990, 42, 4940; Phys Rev B 1991, 44, 1446(E).

25. Engel, E.; Vosko, S. H. Phys Rev B 1994, 50, (10), 498.

26. Springer, M.; Svendsen, P. S.; von Barth, U. Phys Rev B 1996, 54, (17), 392; Svendsen, P. S.; von Barth, U. Phys Rev B 1996, 54, (17), 402.

27. Becke, A. D. J Chem Phys 1986, 84, 4524.

28. Becke, A. D. Phys Rev A 1988, 38, 3098.

29. Lee, C.; Yang, W.; Parr, R. G. Phys Rev B 1988, 37, 785.

30. Langreth, D. C.; Mehl, M. J. Phys Rev Lett 1981, 47, 446.

31. Perdew, J. P.; Wang, Y. Phys Rev B 1986, 33, 8800; 1989, 40, 3399(E).

32. Perdew, J. P. Phys Rev B 1986, 33, 8822; 1986, 34, 7406(E).

33. Perdew, J. P. In P. Ziesche and H. Eschrig (Eds.), Electronic Structure of Solids 1991; Akademie: Berlin, 1991, p. 11.

34. Perdew, J. P.; Burke, K.; Ernzerhof, M. Phys Rev Lett 1996, 77, 3865; 1997, 78, 1396(E).
35. Bagno, P.; Jepsen, O.; Gunnarsson, O. Phys Rev B 1989, 40, 1997.

36. Perdew, J. P.; Chevary, J. A.; Vosko, S. H.; Jackson, K. A.; Pederson, M. R.; Singh, D. J.; Fiolhais, C. Phys Rev B 1992, 46, 6671 .

37. Becke, A. D. J Chem Phys 1992, 96, 2155.

38. Johnson, B. G.; Gill, P. M. W.; Pople, J. A. J Chem Phys 1993, 98, 5612.

39. Barbiellini, B.; Moroni, E. G.; Jarlborg, T. J Phys Condens Matter 1990, 2, 7597; Helv Phys Acta 1991, 64, 164.

40. Leung, T. C.; Chan, C. T.; Harmon, B. N. Phys Rev B 1991, 44, 2923.

41. Singh, D. J.; Ashkenazi, J. Phys Rev B 1992, 46, 11570.

42. Garcia, A.; Elsässer, C.; Zhu, J.; Louie, S. G.; Cohen, M. L. Phys Rev B 1992, 46, 9829; 1993, 47 4150(E).

43. Dufek, P.; Blaha, P.; Schwarz, K. Phys Rev B 1994, 50, 7279.

44. Schmid, R. N.; Engel, E.; Dreizler, R. M.; Blaha, P.; Schwarz, K. Adv Quant Chem (in press).

45. Engel, E.; Vosko, S. H. Phys Rev B 1993, 47, 13164.

46. Sahni, V.; Gruenebaum, J.; Perdew, J. P. Phys Rev B 1982, 26,4371 .

47. Langreth, D. C.; Mehl, M. J. Phys. Rev. B 1983, 28, 1809.

48. Sharp, R. T.; Horton, G. K. Phys Rev 1953, 90, 317.

49. Talman, J. D.; Shadwick W. F. Phys Rev A 1976, 14, 36.

50. Krieger, J. B.; Li, Y.; Iafrate, G. J. Phys Lett 1990, 146A, 256.

51. Norman, M. R.; Koelling, D. D. Phys Rev B 1984, 30, 5530.

52. Li, Y.; Krieger, J. B.; Norman, M. R.; Iafrate, G. J. Phys Rev B 1991, 44, 10437.

53. Chen, J.; Krieger, J. B.; Li, Y.; and Iafrate, G. J. Phys Rev A 1996, 54, 3939.

54. Tong, X.-M; Chu, S. I. Phys Rev A 1997, 55, 3406.

55. Colle, R.; Salvetti, O. Theoret Chim Acta 1975, 37, 329.

56. Grabo, T.; Gross, E. K. U. Chem Phys Lett 1995, 240, 141.

57. Harris, J.; Jones, R. O. J Phys F 1974, 4, 1170.

58. Sham, L. J. Phys Rev B 1985, 32, 3876.

59. Görling, A.; Levy, M. Phys Rev A 1994, 50, 196.

60. Ullrich, C. A.; Gossmann, U. J.; Gross, E. K. U. Phys Rev Lett 1995, 74, 872; van Leeuwen, R. Phys Rev Lett 1996, 76, 3610; Görling, A. Phys Rev A 1997, 55, 2630.

61. Krieger, J. B.; Li, Y.; Iafrate, G. J. Phys Rev A 1992, 45, 101. 62. Krieger, J. B.; Li, Y.; Iafrate, G. J. Phys Rev A 1992, 46, 5453.

63. Krieger, J. B.; Li, Y.; Iafrate, G. J. Phys Rev A 1993, 47, 165.

64. Engel, E.; Vosko, S. H. Phys Rev A 1993, 47, 2800.

65. Kotani, T. Phys Rev B 1994, 50, 14816; Phys Rev Lett 1995, 74, 2989; Kotani, T.; Akai, H. Phys Rev B 1996, 54, 16502.

66. Bylander, D. M.; Kleinman, L. Phys Rev Lett 1995, 74, 3660; Phys Rev B 1995, 52, 14566; Phys Rev B 1996, 54, 7891; Phys Rev B 1997, 55, 9432.

67. Städele, M.; Majewski, J. A.; Vogl, P.; Görling, A. Phys Rev Lett 1997, 79, 2089.

68. Engel, E.; Keller, S.; Facco Bonetti, A.; Müller, H.; Dreizler, R. M. Phys Rev Lett A 1995, 52, 2750.

69. Engel, E.; Dreizler, R. M. In R. F. Nalewajski (Ed.), Topics in Current Chemistry (Vol. 181); Springer: Berlin, 1996, p. 1.

70. Kreibich, T.; Gross, E. K. U.; Engel, E. Phys Rev A 1998, 57, 138. 
71. Engel, E.; Facco Bonetti, A.; Keller, S.; Andrejkovics, I.; Dreizler, R. M. Phys Rev A 1998, 58, 964.

72. Hohenberg, P.; Kohn, W. Phys Rev 1964, 136B, 864.

73. Engel, E.; Chevary, J. A.; Macdonald, L. D.; Vosko, S. H. Z Phys D 1992, 23, 7.

74. Höck, A.; Engel, E. Phys Rev A (in press).

75. Görling, A. Phys Rev B 1996, 53, 7024.

76. Fetter, A. L.; Walecka, J. D. Quantum Theory of Many-Particle Systems; McGraw-Hill: New York, 1971.

77. Taking into account the explicit form eq. (11) of $v_{H}$, it is possible to eliminate a further class of diagrammatic contributions from eq. (42); i.e., the tadpole diagrams (for details see ref. 71). For the present purpose of a lower order expansion, however, this step is not particularly helpful.

78. Zaremba, E.; Kohn, W. Phys Rev B 1976, 13, 2270.

79. Lein, M. Diploma thesis, Würzburg, 1998.

80. Levy, M.; Perdew, J. P. Phys Rev A 1985, 32, 2010.

81. For two-electron systems the OPM, KLI, and HF groundstate energies coincide due to the fact that $E_{x}$, in this case, only eliminates the self-interaction energy of the two electrons. Nevertheless, the spectra of excited states that are obtained from the multiplicative OPM and the nonlocal HF potential are quite different, which manifests itself in the correlation energies of Table V.
82. Gunnarsson, O.; Jonson, M.; Lundqvist, B. I. Solid State Commun 1977, 24, 765.

83. Alonso, J. A.; Girifalco, L. A. Phys Rev B 1978, 17, 3735.

84. Filippi, C.; Gonze, X.; Umrigar, C. J. In J. Seminario (Ed.), Recent Developments and Applications in Density Functional Theory; Elsevier: Amsterdam, 1996.

85. Ishikawa, Y.; Quiney, H. M. Phys Rev A 1993, 47, 1732.

86. Davidson, E. R.; Hagstrom, S. A.; Chakravorty, S. J.; Meiser Umar, V.; Froese Fischer, C. Phys Rev A 1991, 44, 7071.

87. Freund, D. E.; Huxtable, B. D.; Morgan III, J. D. Phys Rev A 1984, 29, 980.

88. For the present calculations a rather large two-center basis of Hylleraas-type has been employed (for details, see ref. 90), checking carefully that the results are converged at the 0.01-eV level.

89. Suhai, S. Phys Rev B 1994, 50, (14), 791.

90. Engel, E.; Schmitt, U. R.; Lüdde, H.-J.; Toepfer, A.; Wüst, E.; Dreizler, R. M.; Knospe, O.; Schmidt, R.; Chattopadhyay, P. Phys Rev B 1993, 48, 1862.

91. Montgomery, J. A.; Ochterski, J. W.; Petersson, G. A. J Chem Phys 1994, 101, 5900.

92. Kolos, W.; Wolniewicz, L. J Chem Phys 1965, 43, 2429.

93. Patton, D. C.; Porezag, D. V.; Pederson, M. R. Phys Rev B 1997, 55, 7454. 